\title{
ENERGETIC PARTICLE OBSERVATIONS CLOSE TO THE SUN BY SOLAR ORBITER AND PARKER SOLAR PROBE
}

\section{O. E. Malandraki ${ }^{a},{ }^{\star}$, D. J. McComas ${ }^{b}$, J. Rodriguez-Pacheco $^{c}$, N. Schwadron ${ }^{d}$, R. Wimmer-Schweingruber ${ }^{e}$, G. Ho ${ }^{f}$}

${ }^{a}$ National Observatory of Athens, IAASARS

Metaxa \& Vas. Pavlou str., Athens, Greece

${ }^{b}$ Department of Astrophysical Sciences, Princeton University

171 Broadmead, Princeton, NJ, USA

${ }^{c}$ Universidad de Alcalá

Pl. de San Diego, s/n, 28801, Alcalá de Henares, Spain

${ }^{d}$ Department, University, University of New Hampshire 105 Main St, Durham, USA

${ }^{e}$ Institut für Experimentelle und Angewandte Physik, Christian Albrechts-Universität zu Kiel Leibnizstrasse 11, Kiel, Germany

${ }^{f}$ Johns Hopkins University, Applied Physics Laboratory 11100 Johns Hopkins Rd, Laurel, USA

E-mail: omalandenoa.gr, dmccomaseprinceton.edu, fsrodriguez@uah.es, nschwadrondguero.sr.unh.edu, wimmeraphysik.uni-kiel.de, George.Ho@jhuapl. edu

\begin{abstract}
A review of the unprecedented measurements of energetic particles in the near-Sun environment obtained by the Solar Orbiter (SolO) and Parker Solar Probe (PSP) pioneering missions and the exciting new results derived is presented. How the SolO/EPD and PSP/IS $\odot I S$ instrument observations are advancing our current knowledge and understanding of the energetic particle environment close to the Sun (e.g. Solar Energetic Particles, Corotating Interaction Region associated suprathermal ions, Cosmic Rays) is reviewed.
\end{abstract}

37th International Cosmic Ray Conference (ICRC 2021)/

July 12th-23rd, 2021

Online-Berlin, Germany

\section{"Presenter}

(c) Copyright owned by the author(s) under the terms of the Creative Commons 


\section{Introduction}

Energetic particle (EP) populations in the heliosphere range from suprathermal solar wind energies to $10^{20} \mathrm{keV}$ (e.g. [1], [2]). The intensity of Galactic Cosmic Rays (GCRs) can be affected by solar wind perturbations at a given heliospheric location. For example, Interplanetary Coronal Mass Ejections (ICMEs) may cause GCR depressions known as Forbush Decreases (FDs). The passage of corotating high-speed solar wind streams (HSSs) may also produce GCR depressions (e.g. [3], [4]) also called recurrent FDs by some authors. Such decreases of the GCR flux are produced by the strong magnetic field structures within ICMEs and HSSs that act as barriers for the propagation of GCRs. In the past, the study of FDs was mainly based on data from neutron monitors on the surface of the Earth, but nowadays, GCR measurements suitable for FD studies are also available from many spacecraft $(\mathrm{s} / \mathrm{c})$ in the near-Earth space as well as on other solar system bodies (e.g. [5], [6], [7], [8], [9]). Anomalous Cosmic Rays (ACRs) originate from interstellar neutrals entering the outer heliosphere, followed by ionization and acceleration that is presumably in association with the termination shock, and penetrate into the inner heliosphere where their intensity is reduced by solar modulation (e.g. [10]) Although well studied at $1 \mathrm{AU}$ and beyond, there have been very few observations of ACRs inside 1 AU and complete lack of measurements in regions exceptionally close to the Sun.

Solar Energetic Particles (SEPs) from suprathermal (few keV) up to relativistic (few $\mathrm{GeV}$ ) energies are emitted from the Sun in association with solar flares and Coronal Mass Ejections (CME)-driven shock waves. Transient IP shocks at $\sim 1$ AU are occasionally accompanied by an enhancement in the intensity of energetic ions above $\sim 0.05 \mathrm{MeV} /$ nucleon, refereed to as an Energetic Storm Particle (ESP) event (e.g. [11], [12]). SEP radiation storms may have durations from a period of hours to days or even weeks and have a large range of energy spectrum profiles. These events pose a threat to modern technology strongly relying on $\mathrm{s} / \mathrm{c}$ and are a serious radiation hazard to humans in space, and additionally of concern for avionics and commercial aviation in extreme circumstances ([13], [14]). Our knowledge of the origin, acceleration and transport of SEPs from near the Sun through the Interplanetary Space (IP) has exhibited immense progress in the last 40 years as more sophisticated instrumentation and modeling capabilities have become available ([12], [15], [16]). However, many puzzles remained unsolved primarily due to the scarcity of in situ measurements well inside 1 AU [12]. Figure 1 shows a clear example of the need for SEP observations in the inner heliosphere. The Helios $1 \mathrm{~s} / \mathrm{c}$ located at $\sim 0.4$ AU detected

Figure 1: $\mathrm{e}^{-}$and He time profiled from Helios 1 (0.3 AU) and IMP-8 (1 AU) during 5 impulsive SEP event in 1980 [17]. Magnetic connections to the flare site are indicated at upper right. Helios 1 observed 5 injections that had merged into a single event by the time they reached IMP-8.

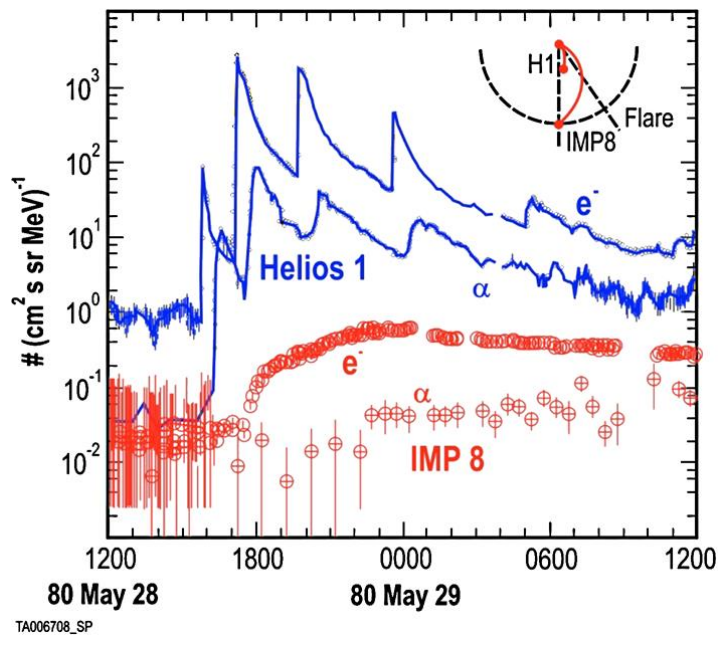


at least 5 separate impulsive-like injections of electrons (e $\mathrm{e}^{-}$) and $\mathrm{He}$ ions, while IMP 8 at $1 \mathrm{AU}$ observed dramatically different time-intensity profiles. This indicated that scattering and diffusion during transport between $0.4 \mathrm{AU}$ to Earth orbit smeared out the time profiles at $1 \mathrm{AU}$, registering a single particle event rather that at least 5, as seen at Helios 1.

EP increases associated with stream interaction regions (SIRs) have been studied for decades. SIRs become Corotating Interaction Regions (CIRs) once they have persisted for at least one solar rotation. When fast coronal streams follow those of the slow solar wind, a compression region forms as the fast solar wind piles up behind the slower one. The compression strengthens as it propagates outward from the Sun, often steepening into a forward-reverse shock pair a few AU from the Sun. The common interpretation of CIR-related events is that the EPs accelerated at these distant shocks propagate back inward toward the Sun, and are observed as the corotating compression region passes over the observer ([3], [4]). [18] provided this traditional model of particle acceleration for CIR-associated EP events, which at sufficiently large distances from the source region predicts a spectrum roll over at low energies, as those particles are unable to penetrate far enough into the inner heliosphere to reach the observer. However, recent studies suggest that additional acceleration could be a result of compressive acceleration along the CIR interface prior to the formation of shocks at the CIR boundaries (e.g. [19], [20]). Most of our knowledge of CIR events comes from observations near $1 \mathrm{AU}$ or beyond. However, the Helios s/c observed several CIR events, including five inside 0.6 AU [21]. Additional observations, particularly those within $1 \mathrm{AU}$ for comparison, are needed to further investigate the energization and transport effects of CIR-associated ions.

Two ongoing pioneering and highly complementary missions are collecting unprecedented in situ measurements of EPs in the near-Sun environment. Parker Solar Probe (PSP) [22] was launched on 2018 August 12. Through a series of Venus flybys, the perihelion of the orbits will gradually be reduced to reach $<10 \mathrm{R}_{\mathrm{S}}(<0.047 \mathrm{AU})$ by late 2024. Onboard PSP the instrument

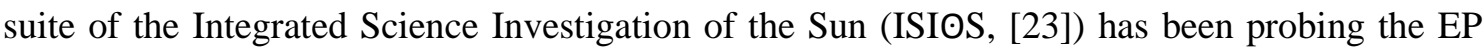
environment near the Sun via the Energetic Particle Instrument-Low (EPI-Lo) and the Energetic Particle Instrument-High (EPI-Hi). EPI-Lo [24] comprises 80 time-of-flight apertures providing particle observations from $20 \mathrm{keV} /$ nuc to $1.5 \mathrm{MeV} / \mathrm{nuc}$ over $2 \pi$ steradians. EPI-Hi [25] encompasses three telescopes of stacked solid-state detectors, utilizing the standard $\mathrm{dE} / \mathrm{dx}$ versus residual energy technique to measure ions from $\sim 1$ to $>100 \mathrm{MeV} /$ nuc and $\mathrm{e}^{-}$from $\sim 0.5$ to $6 \mathrm{MeV}$. Solar Orbiter (SolO) [26], an ESA-led mission with strong NASA participation, was launched on 2020 February 10. Making in-situ measurements close to the Sun and observing the Sun remotely from outside the ecliptic plane are two fundamental drivers for the mission, which will approach the Sun to as close as $0.28 \mathrm{AU}$ and reach heliographic latitudes of up to $33^{\circ}$. The Energetic Particle Detector (EPD) [27] onboard SolO is a key instrument for measuring EPs and solving the riddle of how the Sun accelerates particles to relativistic energies and how these propagate from their acceleration site to fill the heliosphere. EPD consists of 4 individual sensors. The Supra-Thermal Electron Proton sensor (STEP) measures $\mathrm{e}^{-}$and ions between $4 \mathrm{keV}$ ( $\sim 6 \mathrm{keV}$ for ions) and $80 \mathrm{keV}$ with high pitch angle resolution around the direction of nominal magnetic connection to the Sun. The Electron Proton Telescope (EPT) measures e- and ions between $25 \mathrm{keV}$ and $400 \mathrm{keV}$ (6.9 keV and beyond for ions) in 4 look directions. Neither STEP nor EPT discriminate between different ion species. HET measures e $\mathrm{e}^{-}$and ions from $300 \mathrm{keV}$ to $30 \mathrm{MeV}$ and $6.8 \mathrm{MeV}$ to $>100 \mathrm{MeV} /$ nuc. Finally, the Suprathermal Ion Spectrograph (SIS) measures the elemental and isotopic 
composition of ions in the energy range from $\sim 14 \mathrm{keV} /$ nuc to $20.5 \mathrm{MeV} /$ nuc. How the SolO/EPD and PSP/IS@IS instrument observations are advancing our current knowledge and understanding of the EP environment close to the Sun (SEPs, CIR-associated suprathermal ions, Cosmic Rays CRs) is reviewed.

\section{Solar Energetic Particle Observations in the Inner Heliosphere}

An overview of the IS๑IS observations over PSP's first two orbits is presented in Figure 2 [28]. Higher (1-2 MeV) and lower energy (30-200 keV) $\mathrm{H}^{+}$ion count rates are plotted on the outside and inside of the orbital trajectory, respectively. A number of interesting EP events were observed by IS๑IS over a range of radial distances during the first two PSP orbits including CIR/SIR-related events (intervals a and b), events related to ICMEs (interval c) and impulsive SEP events driven by acceleration near the Sun (interval d).

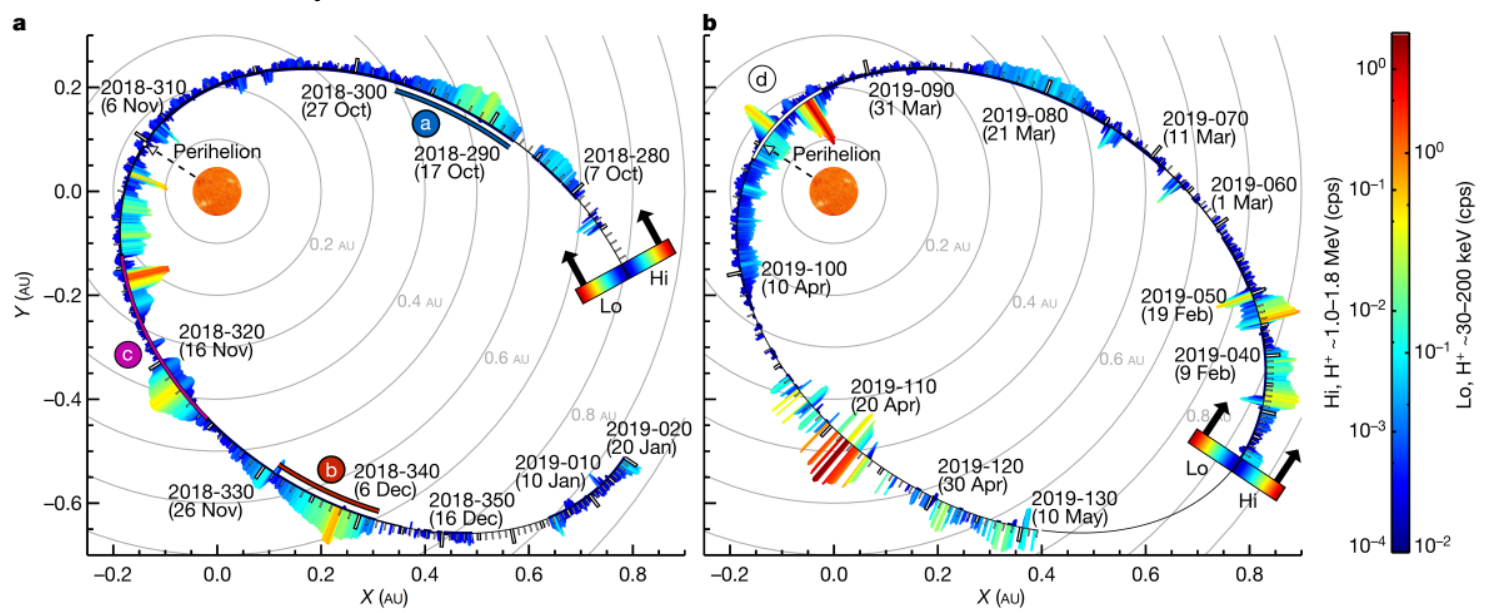

Figure 2: Observations of EPs during PSP orbits 1 and 2. Intensifications indicate EP events, with some seen only at higher energies, some only at lower energies and other simultaneously across the combined energy range. From [28].

Figure 3 shows the events in interval c, including low-energy ions ahead of a CME (Figure $3 \mathrm{~b}, \mathrm{f}, \mathrm{g}$ ), the passage of a compression wave after it (Figure 3c) and a subsequent higher-energy particle event (Figure 3a). An SEP event starting early on 2018-315, is observed only at low energies, extending to the arrival of an ICME at PSP on 2018-316. The event is highly anisotropic (Figure 3f) with EPs streaming outward from the Sun and exhibits velocity dispersion. From the time-energy slope (Figure 3g) a particle path length is estimated longer than that of Parker spiral from PSP at $\sim 0.25 \mathrm{AU}$, possibly explained by a longer pathlength associated with magnetic field 'switchbacks' observed by PSP [29]. The white light coronagraph observations on NASA's Solar Terrestrial Relations Observatory A (STA) s/c indicate that the SEP-associated CME erupted at $3 R_{\mathrm{S}}$ off the east limb of the Sun (as viewed from STA) at $\sim 18: 00$ UT on 2018-314 and was roughly propagating in the direction of PSP. The CME was moving slowly ( $<400 \mathrm{~km} / \mathrm{s})$ from the Sun to PSP as derived by STA imaging. Early modeling indicates there was no shock magnetically connected to the s/c. But a quasi-perpendicular (Q-perp) sub-critical shock could have formed over an extended region of the flux rope and may have accelerated the $\mathrm{p}^{+}$measured [28]. It is noteworthy that this SEP event was not observed at $1 \mathrm{AU}$, so such small events may only be observable near the Sun and thus more common than previously thought [28]. 
Figure 3: (a) Time series of primarily proton $\left(\mathrm{p}^{+}\right.$) flux at $>1 \mathrm{MeV}$, (b) $\mathrm{H}+$ flux around 30-500 keV, (c) solar wind density and (d) radial speed, (e) magnetic field vector and magnitude over interval c. (f)-(h) Magnification of the dispersive SEP event and CME for $\mathrm{H}+$ flux around $30-500 \mathrm{keV}$. From [28].

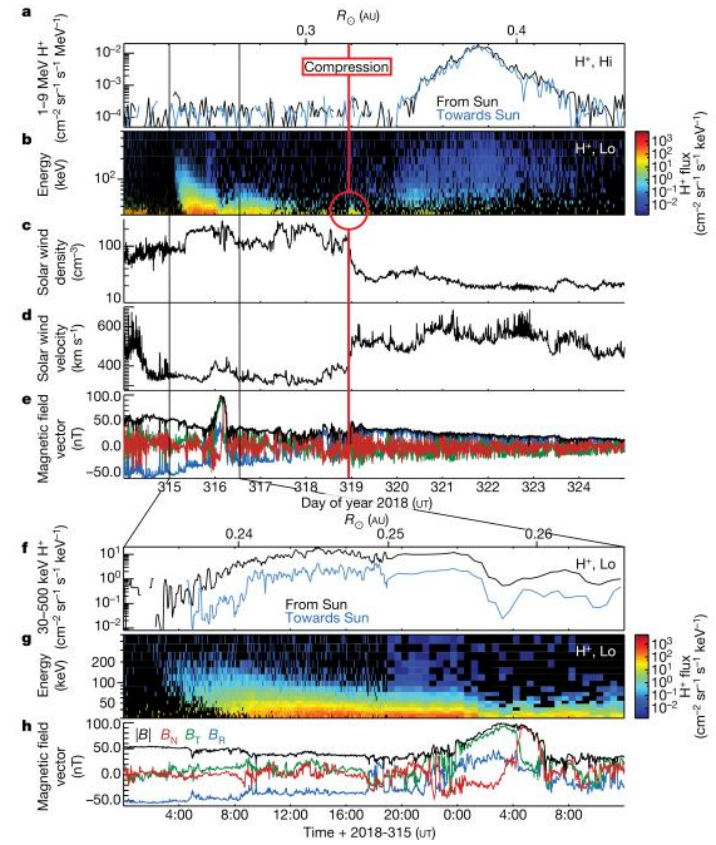

[30] further analyzed this event and found that the onset time of the earliest arriving higher energy ions is consistent with them originating at the CME, when it was at 7.4 $\mathrm{R}_{\mathrm{s}}$ and moving without scattering to PSP, with a pathlegnth equal to $0.21 \mathrm{AU}$. In contrast, ions below $\sim 50 \mathrm{keV}$ arrive later, by about 2-4 $\mathrm{hr}$ compared with the time expected from scatter-free transport. The derived pathlength of these ions ranges from 0.32 to $0.38 \mathrm{AU}$, nearly twice that of the high-energy ions. Giacalone also presented numerical solutions of the Parker transport equation [31] to model and fit the observed ion fluxes (30-300 keV/nuc), assuming a source comoving with the CME. Their model agrees well with the observations (Figure 4). The parallel diffusion coefficient that best fit the data was found to have the form $\kappa=2.7 \times 10^{17}\left(\mathrm{r} / \mathrm{r}_{0}\right)^{2}\left(\mathrm{E} / \mathrm{E}_{0}\right)^{9 / 8}$, where $\mathrm{r}_{0}=7.4 \mathrm{R}_{\mathrm{s}}$ and $\mathrm{E}_{0}=10 \mathrm{keV}$. [30] concluded that the SEP event is consistent with ion acceleration at a weak shock driven briefly by the CME close to the Sun, which later dissipated before arriving at PSP, followed by the transport of ions in the IMF. This event is unique since it is the closest to the Sun in which a CME-related SEP event has been observed. Alternatively, a 'pressure cooker' mechanism found to accelerate particles in planetary auroras may also operate in the solar corona, and has been proposed as a possible SEP accelerator [32].

Very near perihelion ( $\sim 35 \mathrm{R}_{\mathrm{s}}$ ) during PSP Orbit 2 (interval $\mathrm{d}$ in Figure 2) IS๑IS observed a unique pair of SEP events. The first event on 2019-092 (April 2) was a low energy dispersive event whereas the second event on 2019-094 (April 4) was marked by a substantial increase in ions with > $1 \mathrm{MeV}$ energy (see Figure 4 of [28]). Both events exhibit strong, persistent magnetic field-aligned ions streaming away from the Sun. [33] focused on the study of the April 4 SEP event analyzing EPI-Hi data from both the A and B apertures of the Low Energy Telescope LET1. Following background subtraction the $\mathrm{He} / \mathrm{H}$ ratio was found to be $0.052 \pm 0.002$, well determined at 1-2 MeV/nuc, which is higher than the typical value found in SEPs [34] and matches that of $0.052 \pm 0.005$ measured spectroscopically in the corona [35]. Absence of heavy ions relative to He was observed suggesting that the event may be depleted in heavy elements compared with averaged SEP elemental composition. Figure 5 presents time profiles of $1.12-5.66 \mathrm{MeV} \mathrm{p}^{+}$in both LET-A and LET-B. The entire event is very brief, lasting only $\sim 15 \mathrm{hr}$, and takes $\sim 4 \mathrm{hr}$ from onset to reach peak intensities. The SEP event is highly anisotropic. Near the peak of the event, the 
LET-A intensities are a factor of $\sim 30$ times greater than those in LET-B. The very solar quiet conditions made the identification of the solar origins quite straightforward. STA and PSP were only $35^{\circ}$ apart in longitude and $1^{\circ}$ apart in latitude. The STA Extreme UltraViolet Imager (EUVI) images show small Ha surges in the Active Region (AR) 12738, which is the only AR visible to STA on the disk when it rotated into view of Earth a few days later, $\sim 80^{\circ}$ east of the nominal magnetic PSP footpoint, temporally associated with type III emission detected by the FIELDS/RFS detector and the EPI-Hi EPs. This suggests that the field lines expanded over a wide longitudinal range between the AR in the photosphere and the corona. [33] pointed out that the very existence of this SEP event, during an exceptionally quiet solar minimum and completely undetectable in situ at $1 \mathrm{AU}$, raises the possibility that such events are more common than one might expect, possibly providing an important source of seed particles available for acceleration in larger SEP events.

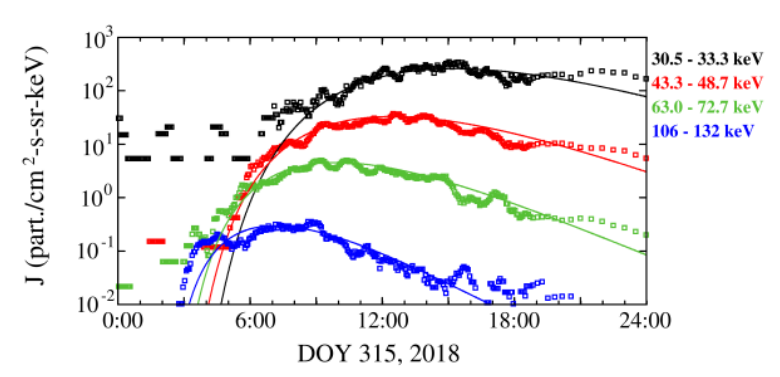

Figure 4: Model calculation (curves) compared with observed EP fluxes (symbols). From [30].

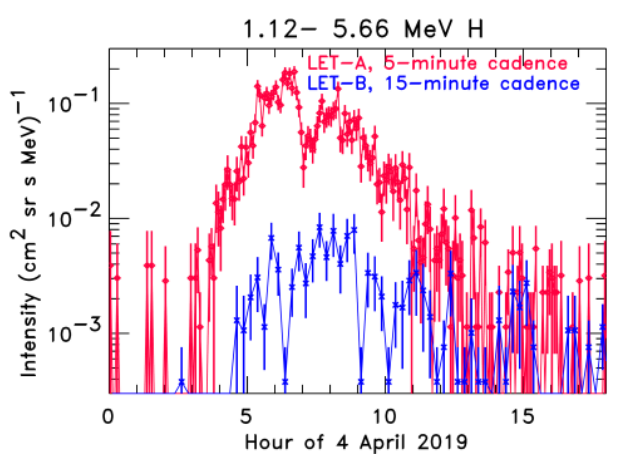

Figure 5: Time profiles of 1.12-5.66 MeV $\mathrm{H}+$ from EPI-Hi/LET1. From [33].

The same AR 12738 produced a series of SEP events in 2019 April 20-21, during the PSP Orbit 2, when PSP was located near 0.48 AU from the Sun [36]. Figure 6, left, panels (a) and (b) show EP fluxes observed by IS $\odot$ IS during the period from 2019 April 18 (Day of Year, DOY 108) to 24 (DOY 114). Due to satellite contact including high-speed data transfers, PSP instruments were operational only intermittently during this period. Four CMEs were released from the Sun on April 18, 20, 21 and 22. The top label shows the radial distance of the PSP s/c, whereas the middle labels denote the start times of the CME events as observed by STEREO and LASCO. The CME released on April 20 overtook the PSP s/c on April 21 near 16:00 UT when the IS $\odot$ IS instruments, solar wind instruments (SWEAP) and magnetic field instruments (FIELDS) were powered on. [36] argued that the observed gradient in the radial speed of the solar wind speed along with the rise in the thermal speed suggests that a compressional plasma structure leading the ICME passed by the s/c. The solar wind plasma ahead of the ICME contained strong enhancements in EP particle fluxes that appear to have been compressed and accelerated within the sheath ahead of the CME. The large radial component of the magnetic field [36] observed throughout the ICME passage makes it unlikely that a flux rope passed over the PSP s/c. Modeling of the propagation of the CME released on April 20 from the Sun using the ENLIL model showed that the location of PSP is on the flank of the CME which is consistent with plasma compression during the passage of the ICME without the accompanying signature of a flux rope [36].

Energetic $\mathrm{e}^{-}$are often nearly scatter-free and provide an unambiguous identification of EP seed populations. During this period PSP was on Parker spiral field lines less than $25^{\circ}$ from the near-Earth s/c. Thus, the Advanced Composition Explorer (ACE) s/c located at the Lagrangian 


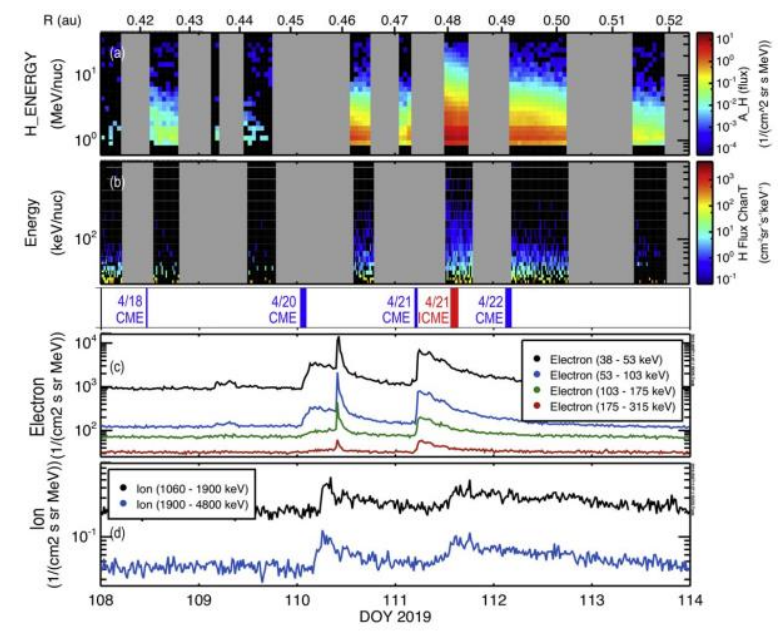

Figure 6: IS $\odot$ IS EP fluxes (panels (a) and (b), outward along the Parker spiral) together with spin and 20-minute averaged $\mathrm{e}^{-}$and ion intensities (panels (c) and (d)) by ACE/EPAM from 2019 April 18-24. From [36].

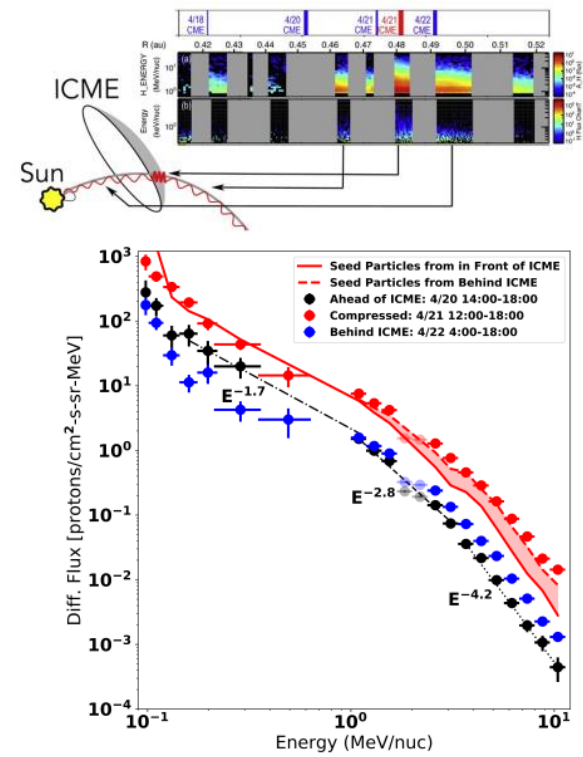

Figure 7: (top) Schematic of the ICME driving a compression region and EP populations observed. (bottom) Observed IS $\odot$ IS EP fluxes plotted together with predictions. Adapted from [36].

L1 point was close to being magnetically connected to PSP. Figure 6, left, panel (c) shows an overview of the 20-minute and spin-averaged differential intensities of four $\mathrm{e}^{-}$channels (DE1DE4: $38-315 \mathrm{keV}$ ) and panel (d) of the 1.1-4.9 MeV energetic ions measured by the Electron, Proton and Alpha Monitor (EPAM) experiment on ACE. On April 20 and 21, two near-relativistic (NR) prompt $\mathrm{e}^{-}$events were observed in which particles accelerated in a magnetically wellconnected solar source region arrive abruptly at the s/c [37]. Both events on April 20 and 21 showed strong ${ }^{3} \mathrm{He}$ enhancements [38] observed by IS๑IS, which definitively support the concept that EP seed populations contained flare-accelerated material [36]. Modeling of compression and acceleration of seed populations swept up into the compression driven by the ICME is carried out by [36]. Figure 7, bottom presents the ion spectra averaged before, during, and after the ICME passage (Figure 7, top) plotted together with predictions. The fluxes predicted by the compression mechanism (red curves in Figure 7, bottom) are generally similar to observations within the ICME-driven compression. Below $1 \mathrm{MeV}$, the IS $\odot$ IS observations show a very hard $\mathrm{E}^{-1.7}$ energy spectrum, which is close to the $\mathrm{E}^{-1.5}$ limit of possible stationary-state plasma distributions out of equilibrium ([39], [40]), which suggests that suprathermal EPs may play a more fundamental role for the pressure and heating of the solar wind. [36] conclude that during this period PSP was at the right place and at the right time to reveal the enhancement of EP seed populations observed within the CME-driven compression which could precondition the EP acceleration process at distances farther out in the heliosphere with compressions steepening into shocks.

During the PSP Orbit 4, [41] analyzed a CME that passed over PSP on January 20, 2020 when the $\mathrm{s} / \mathrm{c}$ was at $0.32 \mathrm{AU}$ from the Sun, an event which is similar to the low energy EP event associated with a CME measured by PSP on November 11, 2018 ([28], [31], [32]). Figure 8 shows IS $\odot$ IS spectrogram observations for different look directions viewed by EPI-Lo, showing the 
evolution of EP anisotropy during the course of the event. A strong EP signature is first seen midway during January 19, highly anisotropic with EPs mostly coming from the Sun, with strong evidence of velocity dispersion, with features consistent with both a remote source and one close to the Sun. The magnetic field is also nearly radial during this time, facilitating more direct transport of EPs from the Sun. These fluxes cut out at about the same time that the s/c passes through a sector boundary crossing early on January 20, indicating that the connection to the remote EP source has been cut off by the crossing of the heliospheric current sheet (HCS).

Figure 8: Spectrograms showing the evolution of the EP anisotropy during the course of the event (from top to bottom: omni-directional, from Sun, toward Sun, and transverse). The bottom panel shows the magnetic field in RTN coordinates. A strong influx of EP propagating toward the Sun is highlighted in yellow, while dropouts in flux are highlighted in orange. From [41].

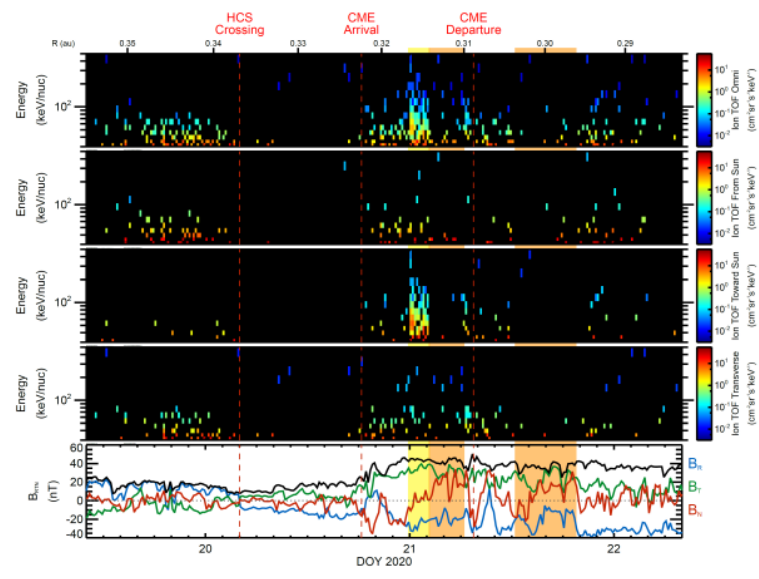

As shown in Figure 8, as the CME passes over the s/c, the normal component of the magnetic field rotated from south to north, coinciding with a very strong influx of EPs moving toward the Sun (highlighted in yellow), lasting only a few hours [41]. As the normal component reaches its northern apex, the flux of EPs in all directions largely drops out (highlighted in orange), lasting for a few hours. A second dropout period is seen later in the day, after the passage of the CME, when the normal component of the field is again in the northward direction (also shown in orange). As [41] highlighted the magnetic field remains within the field of view (FOV) of the instrument during these dropouts, so they seem to be related to a disconnection from the source rather than the rotation of a field-aligned beam of EPs rotating out of the instrument FOV. During this time PSP was radially aligned with STA which measured the same CME when it passed 1 AU. The magnetic field measurements at both $\mathrm{s} / \mathrm{c}$ are found to be remarkably similar, indicating that the $\mathrm{s} / \mathrm{c}$ are likely encountering the same portion of the magnetic structure that has not evolved significantly in transit. However, the EP population at STA is observed to be more uniform throughout the event in contrast to the strong variations seen at PSP, showing how transport effects have acted on the EP populations and obscured the detailed properties present earlier in the development of the CME close to the Sun. [41] offered a unique case study of how the CMEassociated EP populations evolve as it propagates outward through the heliosphere.

During Orbit 5 just before perihelion PSP detected a series of 6 SEP events in May-June 2020 at distances from 0.45-0.3 AU [42]. Figure 9, left shows IS $\odot$ IS observations during this time period where $\mathrm{p}^{+}$intensities are shown as a function of energy and time (from HETA, top panel; LETA, $2^{\text {nd }}$ panel; and EPI-Lo, $3^{\text {rd }}$ panel), helium intensities (from LETA, $4^{\text {th }}$ panel) and $\mathrm{e}^{-}$ count rates (from HETB, bottom panel). The six events are most clearly seen in the LETA $\mathrm{p}^{+}$

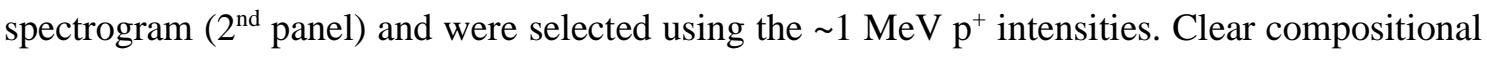
differences between the six events can be seen qualitatively. While all six events are evident in the $\mathrm{p}^{+}$, only events 3 and 5 appear to have a clear $\mathrm{e}^{-}$signal at the EPI-Hi energies. Event 3 appears 

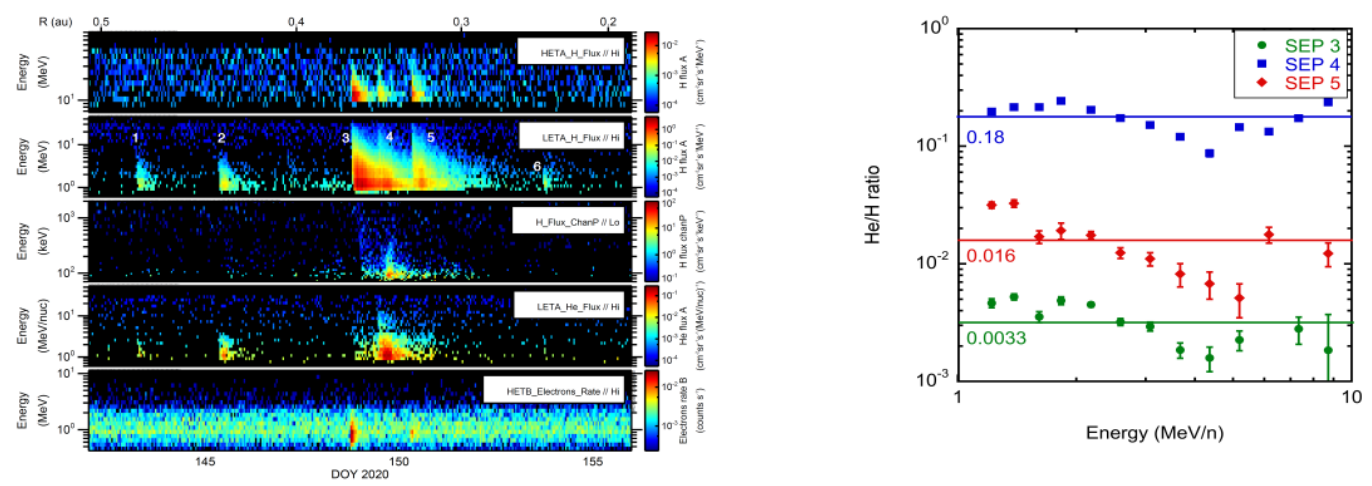

Figure 9. (left) Spectrogram showing the $\mathrm{p}^{+}$intensity vs energy and time from various IS $\odot$ IS detectors (right) $\mathrm{He} / \mathrm{H}$ abundance ratios as a function of energy for events 3, 4 and 5. From [42].

to be the strongest in $\mathrm{p}^{+}$, yet event 4 is the dominant one in He. Within uncertainties [42] found that the $\mathrm{He}$ and $\mathrm{H}$ spectra have similar power law forms with indices ranging from -2.3 to -3.3 . For the 3 largest events, the $\mathrm{He}$ and $\mathrm{H}$ spectra can be used to calculate $\mathrm{He} / \mathrm{H}$ abundance ratios as a function of energy from $\sim 1-10 \mathrm{MeV} /$ nuc (Figure 9, right). The $\mathrm{He} / \mathrm{H}$ ratios are found to be relatively independent; however, the ratios differ substantially with values of $0.0033,0.177$ and 0.016. [42] conclude that these variations are particularly interesting as the 3 larger events are likely a result of similar eruptions from the same AR. [43] concluded that the long apparent pathlength can be explained by a magnetic field line pathlength increase due to field line random walk, and EP transport about the guiding center with a nonzero effective pitch angle.

SolO's inner heliospheric trajectory for the first year of measurements of EPD is shown in Figure 10, presented by [44] from 28 February 2020 to 28 February 2021 with intensities of 54$101 \mathrm{keV} \mathrm{e}^{-}$on the inner side and 124-218 keV ions on the outer side (EPD/EPT detector head). Apart from a low level of background activity (see [45] for an in-depth discussion) and small increases due to CIRs [46], a number of intensity increases can be seen. A list of the most significant EP enhancements as observed by EPT during the $1^{\text {st }}$ year is provided by [44]. Furthermore, updates on EPD as well as additional insights into its operation and data products based on EPD's first year of operation are provided. This article is valuable giving the reader and potential users a good understanding of the EPD data and data products and pointing out possible pitfalls and open issues as well as giving guidelines and solar particle event examples for its usage.

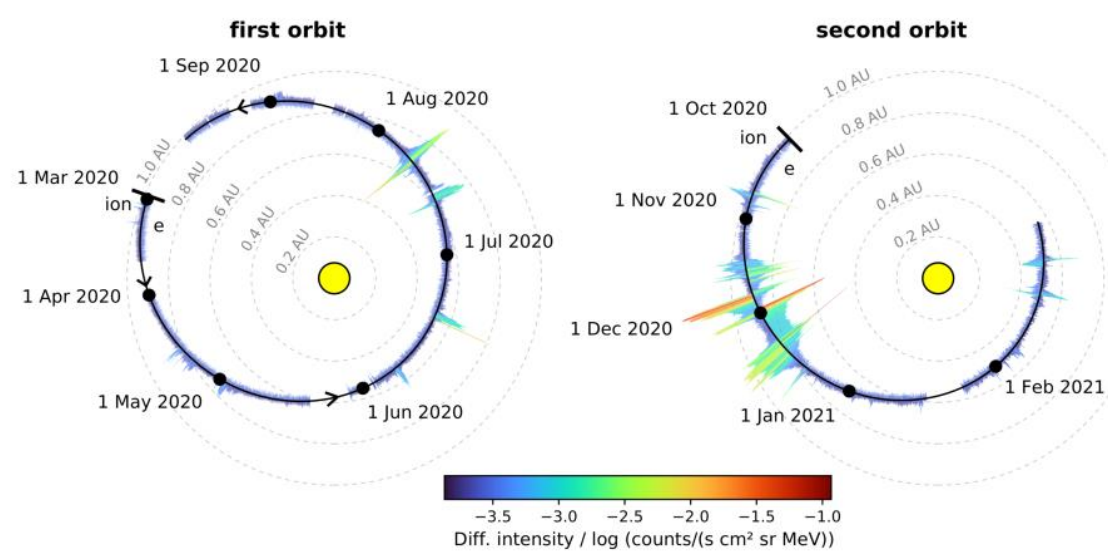

Figure 10. One year's worth of data from EPD/EPT. From [44]. 
[47] studied the first NR solar e- events observed by EPD onboard SolO in July 2020 after its first perihelion. They combined EPD e- observations from $\mathrm{keV}$ energies to the NR range with type III radio bursts from RPW [48] and extreme ultraviolet (EUV) observations from STA and the near-Earth Solar Dynamics Observatory (SDO) to identify the solar origins of the in situ NR $\mathrm{e}^{-}$events. During the period under study, SolO was found to be magnetically connected to the solar backside (as viewed from the Earth). All five $\mathrm{e}^{-}$event increases were found to be clearly associated with type III radio bursts. EUV jets were also found in association with all of them except one. A diversity of time profiles and pitch-angle distributions (PADs) was observed. Figure 11 shows observations and analysis of the July 22, $2020 \mathrm{e}^{-}$event [47]. EPT observed a very anisotropic $\mathrm{e}^{-}$event reaching energies slightly above $100 \mathrm{keV}$ (Figure 11, left). The spike-like time profile showed a sharp onset with clear velocity dispersion, starting at 23:40 UT $\pm 10 \mathrm{~min}$ at 58 $\mathrm{keV}$. This period was clearly observed by STEP at lower energies, which revealed additional spike-like injections preceding and following the event observed by EPT. The EPD pitch-angle coverage was nearly optimal (Figure 11, top right).

The bottom panel of Figure 11, right shows an inverse-speed vs time plot (typically used for velocity dispersion analysis) for the July 22 event, combining STEP and EPT e- data. The color scale represents the intensity, normalized to the maximum value for each energy channel. Two different injections were resolved at low energies, both showing clear velocity dispersion and very narrow time profiles over the whole energy range. The black slanted line is a linear fit of the onset times at each energy bin above $9 \mathrm{keV}$ for the second injection. The onset times are defined with the $2 \sigma$ method (e.g. [49]). The linear fit provides an estimation of the effective path length $\mathrm{L}$ and the solar release time (SRT) at the Sun. The theoretical Parker spiral length connecting to SolO for a solar wind speed of $320 \mathrm{~km} / \mathrm{s}$ is $0.77 \mathrm{AU}$, which is substantially shorter than the value $\mathrm{L}=1.02$
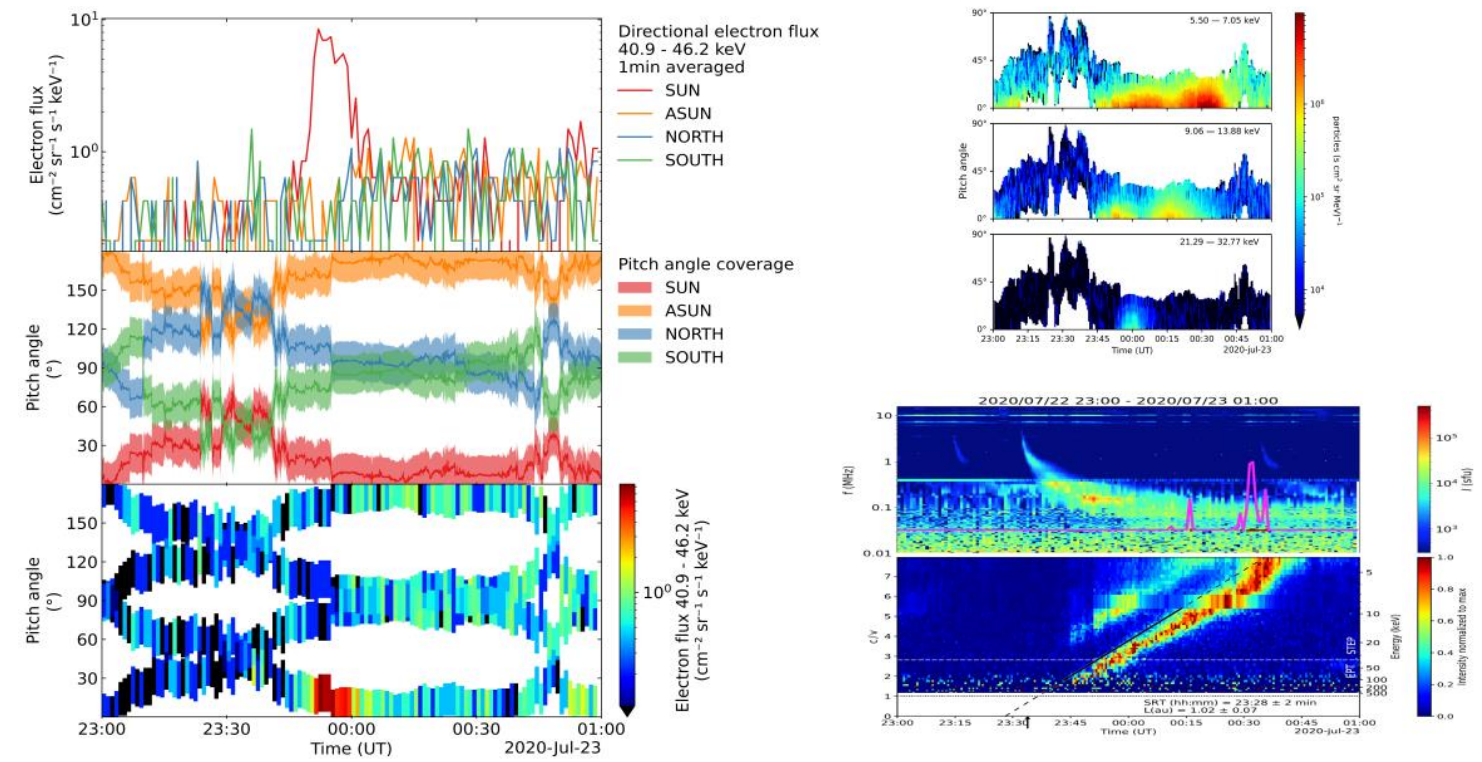

Figure 11. (left) Time evolution of directional $\mathrm{e}^{-}$intensity measurements during the July 22, 2020 event From top to bottom: 40.9-46.2 keV e- intensities observed by the four EPD apertures; pitch-angle coverage for each aperture; PAD with color-coded intensity for the same energy range shown in the first energy panel (top right) e- PAD by STEP at three different energy bands during the July 22 event (bottom right) SolO/RPW radio dynamic spectrum observed in association with the $\mathrm{e}^{-}$event and the $\mathrm{e}^{-} \mathrm{c} / \mathrm{v}$ versus time plot. Adapted from [47]. 
AU obtained from the fit. The black arrow at 23:34 UT marks the SRT shifted by the light propagation time to SolO in order to allow a direct comparison with electromagnetic observations from 0.69 AU. The top panel of Figure 11, bottom right, shows the radio dynamic spectrum observed by SolO/RPW during this period. A type III radio burst was observed by RPW, starting at 23:32 UT, thus the inferred $\mathrm{e}^{-}$release time and the type III radio burst differ only by 2 minutes.

[47] concluded that the spike-like time profiles, prompts onsets and beamed PADs together with the in-situ detection of Langmuir waves by RPW (magenta line in Figure 11, bottom right) support a very good magnetic connection to the source. However, although STA observed a jet at the east solar limb, the nominal magnetic footpoint of SolO along the ideal Parker spiral was located more than $30^{\circ}$ behind the east limb from the STA viewpoint. [47] found good time coincidence of the type III radio burst, the jet, and the inferred $\mathrm{e}^{-}$release time. Further investigation is required to clarify the magnetic connectivity and IP propagation for this $\mathrm{e}^{-}$event.

${ }^{3} \mathrm{He}$-rich SEP events have been associated with EUV jets [50] suggesting acceleration via magnetic reconnection involving field lines open to IP space [51]. These events are highly associated with type III radio bursts. The first ${ }^{3} \mathrm{He}$-rich events observed by $\mathrm{SolO}$ were measured during the s/c's first perihelion pass from 0.52 to $0.96 \mathrm{AU}$ [52] in June-September 2020. Three out of five discrete events reported by [52] have a $0.2-2 \mathrm{MeV} / \mathrm{nuc}{ }^{3} \mathrm{He} /{ }^{4} \mathrm{He}$ above $10 \%$, with a maximum ${ }^{3} \mathrm{He} /{ }^{4} \mathrm{He}$ of 0.61 . [53] reported observations of a relatively long period of ${ }^{3} \mathrm{He}$-rich SEPs, spanning almost 7 days in November 2020, observed by SolO at 0.96 AU. Such a long period may indicate a nearly continuous ${ }^{3} \mathrm{He}$-rich SEP injection into IP space [53].
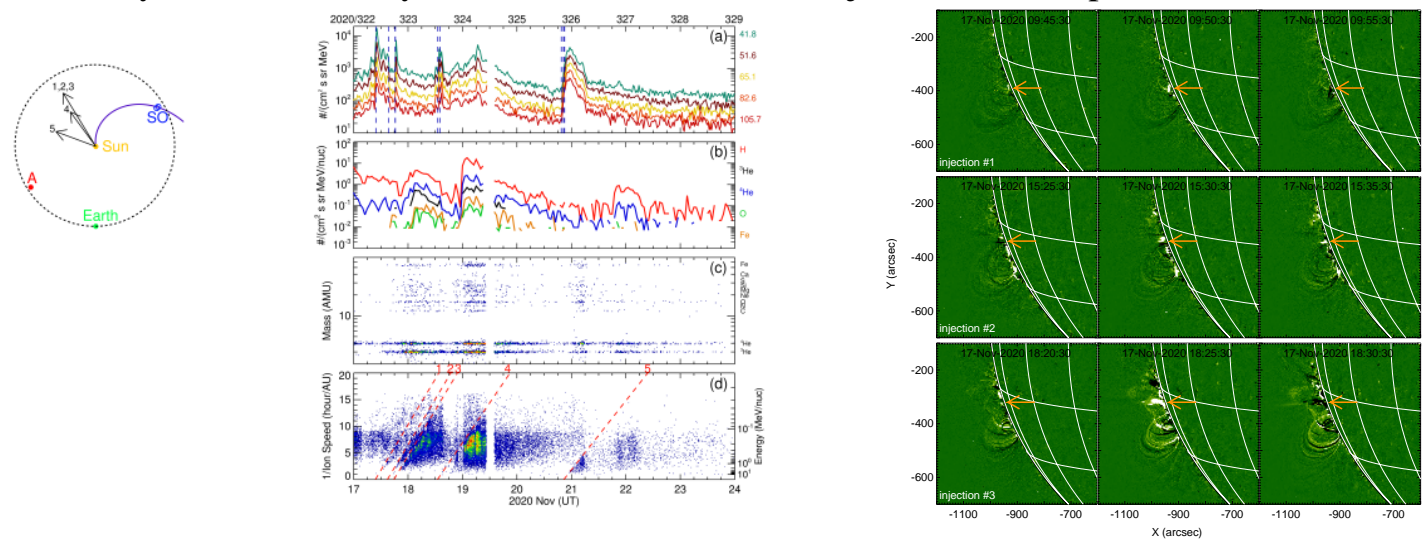

Figure 12. (left) Location of s/c (middle) SolO EP measurements from November 17-23, 2020 (right) STA 195 Å EUV running difference images for injections \#1, \#2, \#3. From [53].

Figure 12, middle presents SolO EPT and SIS measurements from November 17-23, 2020. Panel (a) shows 30-min e- intensities from 41.8-105.7 keV. Panel (b) presents hourly averages of the $0.23-0.32 \mathrm{MeV} / \mathrm{nuc} \mathrm{H},{ }^{3} \mathrm{He},{ }^{4} \mathrm{He}, \mathrm{O}$ and $\mathrm{Fe}$ intensities as measured by both telescopes of the SIS spectrometer. The mass spectrogram in panel (c) shows almost continuous ${ }^{3} \mathrm{He}$ presence from the middle of November 17 through the end of November 23 i.e. for $\sim 6.5$ days. The inverse ionvelocity time spectrogram in panel (d) shows this period consists of five well-resolved ion injections. These injections are identified based on their characteristic triangular pattern in the inverse speed plots. Figure 12, left, shows the location of SolO and STA during the period under investigation. The angular separation between SolO and STA during this period was $180^{\circ}$. The SDO s/c is in orbit around the Earth. The arrows indicate the longitude of the solar source associated with the ion injections. The high-resolution STA imaging observations reveal that the 
injections coincide with EUV jets and brightenings near the east limb, not far from the nominal magnetic connection of SolO. Figure 12, right shows as an example the STA $195 \AA$ EUV running difference images corresponding to injection \#1 (top row), injection \#2 (middle row) and injection \#3 (bottom row). The arrow marks the solar source. [53] concluded that the relatively long period of ${ }^{3} \mathrm{He}$-rich SEPs observed by SolO is related to the recurrent activity (brightenings and jets) in a large and complex group of sunspots in two adjacent ARs, as observed by SDO when the regions rotated in to the Earth's view. Such a configuration may be favorable for the recurrent particle injection in the sense that there may be a long-lived interaction between the negative polarity of one AR and the positive polarity of the neighboring AR, leading to magnetic reconnection. Furthermore, these two ARs produce a longitudinally extended source $\left(\sim 40^{\circ}\right)$ in which s/c may be magnetically connected for a long period as the Sun rotates.

The first widespread SEP event of solar cycle 25 observed on November 29, 2020 was analyzed and discussed by [54], enabling for the first time the comparison and combination of EP observations from four different locations $\leq 1$ AU with a wide longitudinal separation. Figure 13 shows an overview of the EP observations from 2020 November 29 to 2020 December 3 collected by SolO, PSP, STA and by near-Earth s/c. Each panel shows intensity-time profiles of e- (upper portion of the panels) and protons $\left(\mathrm{p}^{+}\right)$(lower portion of the panels) as observed by EPI-Lo (gray) and EPI-Hi (magenta) detectors oof ISIS on board PSP, the EPT (gray) and HET (blue) onboard SolO, the SEPT (gray) and HET (red) on board STA, EPAM (gray) onboard ACE and EPHIN (green) onboard SOHO. The middle right panel shows the s/c locations of PSP (magenta), SolO (blue), STA (red) and near-Earth s/c (green) as seen from the north ecliptic on November 29 13:00 UT, close to the time when the onset of the large SEP event was observed. These s/c covered a longitude span of $\sim 238^{\circ}$ and clearly observed intensity increases of $\mathrm{p}^{+}$at energies $>50 \mathrm{MeV}$ and $\mathrm{e}^{-}$at energies $>1 \mathrm{MeV}$. Nominal Parker spiral IMF lines connecting each s/c with the Sun are also shown calculated using the solar wind speeds measured near the event onset time. Since PSP solar wind data are not available and the solar wind analyzer on SolO/SWA was not operational speed obtained from ENLIL simulations is used instead. The SEP event was associated with an M4.4 soft X-ray flare that occurred at NOAA AR 12790, located just behind the east limb as viewed from Earth (Figure 13). STA/EUVI images at 13:00 UT show that the flare was located at E98S23 (in Stonyhurst Heliographic (HGS) coordinates). The SEP event was associated with the eruption of a fast and relatively wide CME, an EUV-wave expanding in the low corona, and a white-light shock wave observed higher in the corona seen by STA/COR-2 and SOHO/LASCO-C2.

[54] in their first interpretation, inferred the particle release times at the Sun by means of Velocity Dispersion Analysis (VDA) and Time Shift Analysis (TSA) and compared them with the propagation of the EUV wave. Figure 14, left, shows the comparison of the inferred particle release times with the arrival time of the EUV wave at the s/c field line footpoints. The * markers show the angular distance of the EUV wave from the AR. The release times for $\mathrm{e}^{-}$and $\mathrm{p}^{+}$are shown by $\mathrm{x}$ and + markers for each s/c The angular distance of the magnetic footpoint from the $\mathrm{AR}$ for each s/c is shown by the vertical bars, which assume an uncertainty of $\pm 5 \mathrm{deg}$. [54] concluded that a simple scenario where the EP release is only determined by EUV wave propagation is unlikely for this event. The significant anisotropy of the first arriving $\mathrm{e}^{-}$and the detection of Langmuir waves at STA provide evidence that non-nominal magnetic connections are an important element of this widespread event. Furthermore, a prolongation of the path length for $\mathrm{p}^{+}$caused by a turbulent random-walk or by the meandering of field lines in the corona and IP 
space as modeled by [55] would increase the $\mathrm{p}^{+}$path length from those assumed in TSA and would hence reduce the time differences between the $\mathrm{p}^{+}$release times and the EUV wave connection times. [56] have recently investigated this scenario further carrying out more detailed analysis of the CME-driven shock and its particle acceleration efficiency and concluded that the shock does indeed play an important role in the spread of SEPs in this event.

In their second interpretation, [54] compared the observed $\mathrm{e}^{-}$and $\mathrm{p}^{+}$onset delays in the November 2020 event with observations of similar SEP events during solar cycle 24, and considered the implications of a simple interpretation of the observed EP delays. Figure 14, right shows the comparison of $\mathrm{e}^{-}$(top panels) and $\mathrm{p}^{+}$(middle panels) onset delays vs connection angle

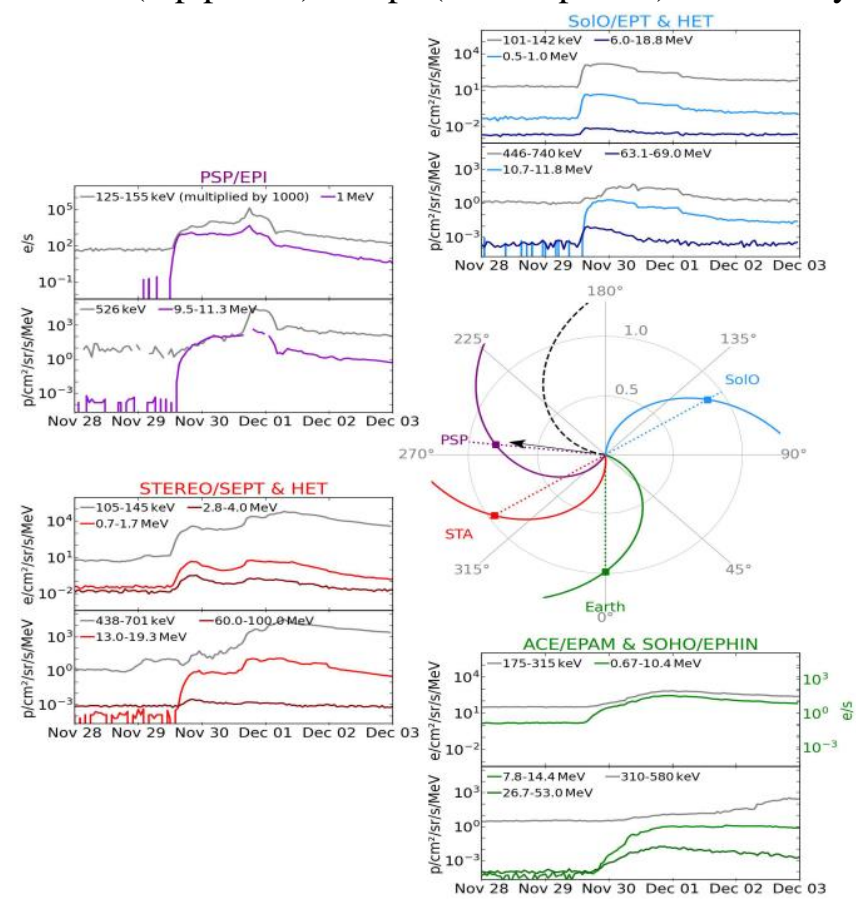

Figure 13. Overview of the 2020 November 29 SEP event. The orbital locations of PSP (magenta), SolO (blue), STA (red) and near-Earth s/c (green) as seen from the north ecliptic are shown as well nominal IMF lines connecting each s/c with the Sun. The black arrow indicates the location of the AR associated with the event. Surrounding panels illustrate hourly-averaged $\mathrm{p}^{+}$ fluxes (lower panels) and e- fluxes (upper panels) observed by the different s/c. From [54].

for the November 2020 SEP event (colored circles indicating the observing s/c as in Figure 13) with those for $\sim 0.7-4 \mathrm{MeV} \mathrm{e}^{-}$and 14-24 MeV $\mathrm{p}^{+}$in the cycle 24 SEP events observed at both STEREO s/c and near the Earth discussed by Richardson et al. 2014 [57] (black circles). It is noteworthy that the particle delays in the November 20 event are similarly ordered by increasing connection angle. They are slightly lower than the average for the cycle 24 events. The bottom panels in Figure 14 show the "source speeds" from the flare to the field line footpoint as a function of connection angle for $\mathrm{e}^{-}$(left) and $\mathrm{p}^{+}$inferred from the November 2020 event onset delays. The different source speeds suggest that the $\mathrm{e}^{-}$and $\mathrm{p}^{+}$onset delays cannot be accounted for by a connection to a single particle source moving away from the solar event near the Sun. Thus, the scenario of efficient cross-field transport of particles could be the dominant process leading to broad longitudinal particle spread at 1 AU. [54] pointed out that a combination of these interpretation scenarios to account for this widespread SEP event cannot be ruled out. 

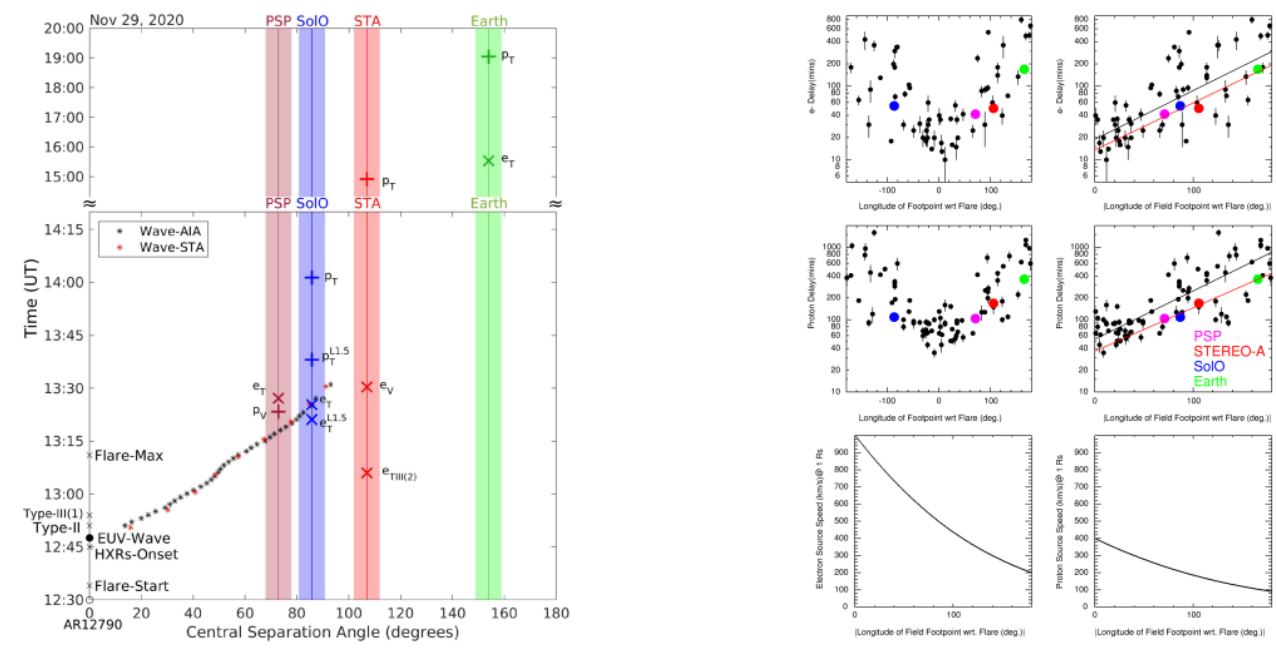

Figure 14. (left) Comparison of the inferred SRT with the EUV wave arrival time at the s/c field line footpoints (right) Alternative interpretation for this widespread SEP event. From [54].

\section{Corotating Interaction Region-associated Suprathermal lons in the Inner Heliosphere}

In the first two orbits of PSP seven particle increases observed by the IS $\odot$ IS/EPI-Hi instrument were identified as SIR events [58]. Several of these events occurred when PSP was inside of $0.65 \mathrm{AU}$ (with two inside $0.5 \mathrm{AU}$ ), adding to the limited catalog of these events observed well inside Earth's orbit, having been previously only measured by Helios [21]. The top panel of Figure 15, left shows the energy and time variation of the seven events as observed by the sunward facing aperture LETA. The events are relatively small with $\mathrm{p}^{+}$increases evident up to only a few $\mathrm{MeV}$ but that last for several days. The magnetic footpoint of the $\mathrm{s} / \mathrm{c}$ was determined by calculating the Parker spiral connecting the Sun and PSP, assuming a solar wind speed of $400 \mathrm{~km}$ $\mathrm{s}^{-1}$. In the bottom panel of Figure 15, left the heliocentric distance of the $\mathrm{s} / \mathrm{c}$ is plotted versus the

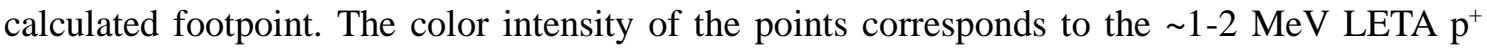
counting rate. Events 1 and 3 have occurred at nearly the same solar footpoint longitude, suggesting these are corotating [28]. Event 7, observed 3.5 months later (2019 March 17), also occurs at the same solar longitude, yet no corresponding particle increases were observed when PSP passed by the same longitude three times in the intervening interval. Thus, this event is considered separate and unrelated to the previous two. Figure 15, right shows a comparison of the $\mathrm{p}^{+}$spectra of the seven events [58]. A power law corresponding to $\mathrm{E}^{-4.5}$ is also shown for reference. Fitting of the spectra result to power-law indices ranging from -4.3 to -6.5 . [59] have determined that the stream interfaces of events 2 and 3 arrive at STA after further corotation.

[58] found that all of the events, with perhaps the exception of event 7 , have softer spectra than the $\mathrm{E}^{-4}$, which is typical for CIR events observed at $1 \mathrm{AU}$ [3]. Whether this is related to the acceleration conditions or the transport conditions is unknown. The He spectra were similarly soft and $\mathrm{He} / \mathrm{H}$ abundance ratios near $1 \mathrm{MeV}$ nuc ${ }^{-1}$, varying somewhat from event to event with values between 0.016 and 0.031 obtained for the events. These ratios are lower than the average $\mathrm{He} / \mathrm{H}$ abundance of CIR events at $1 \mathrm{AU}$. However, they are consistent with the ratio obtained by [60] for CIR events in which the speed of the fast solar wind stream was $\leq 600 \mathrm{~km} \mathrm{~s}^{-1}$, which is the case for nearly all the fast solar wind period observed during the events analyzed. None of the 
events occurred near a clear solar wind shock/compression region and all exhibited EP isotropy and a lack of clear velocity dispersion. This suggests the EP enhancements are not a result of local/near shock acceleration but rather correspond to regions previously filled with EPs sweeping over the s/c. More plausibly, the particles are accelerated at larger distances from the Sun where SIRs may have developed into either shock pairs or at least significant compression regions.
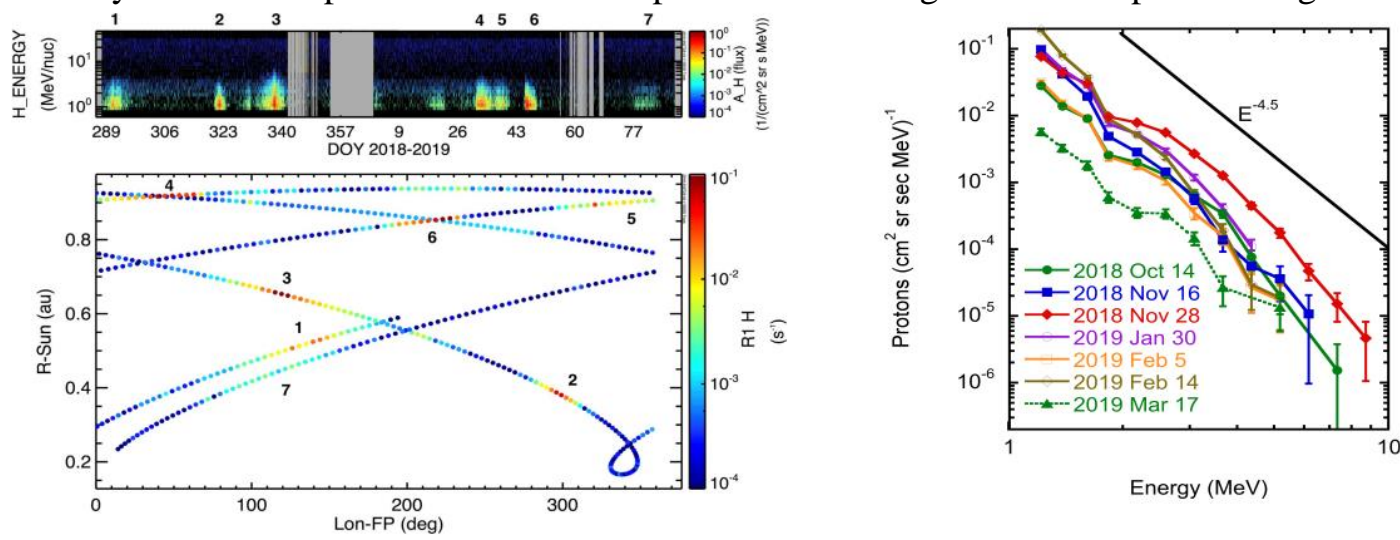

Figure 15. Energetic particle increases associated with SIRs (see text). Adapted from [58].

[61], using IS๑IS/EPI-Lo data, identified six distinct enhancements in the intensities of suprathermal-through-energetic $\left(\sim 0.03-3 \mathrm{MeV} \mathrm{nuc}^{-1}\right)$ He ions associated with CIRs or SIRs during the first two orbits of PSP. Three of these events were also observed by EPI-Hi as reported by [58]. Figure 16 presents the first-ever measurements of suprathermal-through-energetic He ions with energy between $\sim 0.03$ and $2 \mathrm{MeV}$ nuc $^{-1}$ during one intensity enhancement between 2018, DOY 318-325 that was associated with a CIR or SIR when PSP was at $\sim 0.35$ AU following its first perihelion pass. Figure 16, left from top to bottom shows (a): $4 \mathrm{hr}$ averages of the $\sim 0.03-1.99$ $\mathrm{MeV} \mathrm{MeV} \mathrm{nuc}{ }^{-1} \mathrm{He}$ intensities averaged over all 80 EPI-Lo apertures; (b): $4 \mathrm{hr}$ averages of 0.031$0.062 \mathrm{MeV}_{\text {nuc }}{ }^{-1} \mathrm{He}$ intensities averaged all apertures that measure particles flowing in the antisunward, transverse and sunward directions; (c): given by $\gamma=\log \left(\mathrm{j}_{1} / \mathrm{j}_{2}\right) / \log \left(\mathrm{E}_{1} / \mathrm{E}_{2}\right)$, where $\mathrm{j}_{1}$ and $\mathrm{E}_{1}$ are the average intensity and center energy (arithmetic mean) of the lower energy range, and $\mathrm{j}_{2}$ and $\mathrm{E}_{2}$ are the average intensity and center energy, respectively, of the next higher energy range shown in Figure 16(a); (d): the 1/ion speed spectrogram versus time; (e)-(g): 1-minute averages of the magnetic field magnitude from FIELDS and the 1-minute averages of the solar wind density and speed from SWEAP. The latter panels show that the magnetic field magnitude and solar wind density become relatively steady as PSP exits an interaction region and encounters a moderately high speed ( 500 $\left.\mathrm{km} \mathrm{s}^{-1}\right)$ solar wind stream on 2018, DOY 319 [59]. The steady decline in the magnetic field magnitude and the relatively stable, low density through 2018, DOY 324 seen in panels (e) and (f) respectively, indicate that PSP remained inside the rarefaction region throughout this period. Panel (b) shows that the intensities of $\sim 0.031-0.062 \mathrm{MeV} \mathrm{nuc}^{-1} \mathrm{He}$ ions flowing toward the Sun during the peak of the enhancement are either slightly greater or comparable as those flowing in the antisunward and transverse directions. Furthermore, Figure 16(b) shows the late arrival of higher-energy ions as a substantial increase in the counts per bin for ions with the highest speeds between DOY 321 and DOY 322, thereby exhibiting inverse velocity dispersion.

Figure 16, right compares the omnidirectional, event-averaged spectra in all six events [61]. Also included in Figure 16(a), right are data from EPI-Hi/LET as reported by [58], for events \#1, \# 5 and \# 6 above $\sim 1 \mathrm{MeV}$ nuc ${ }^{-1}$. Figure 16(b), right shows the spectra for events \#2, \#3 and \#4 

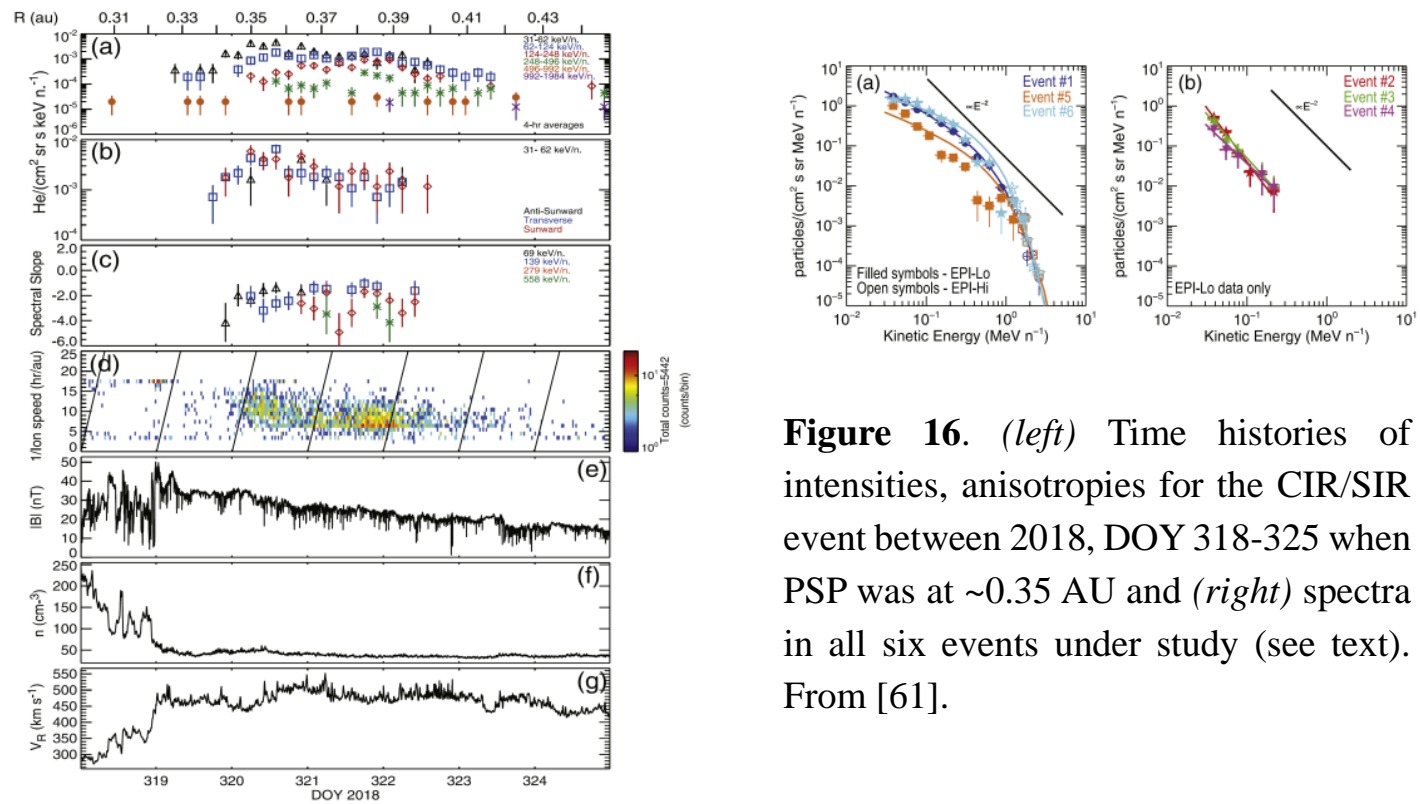

Figure 16. (left) Time histories of intensities, anisotropies for the CIR/SIR event between 2018, DOY 318-325 when PSP was at $\sim 0.35 \mathrm{AU}$ and (right) spectra in all six events under study (see text). From [61].

From EPI-Lo only, as these events are not observed by EPI-Hi. Solid curves in panel (a) are fits to the spectra of the form $j=j_{o} E^{-\gamma} \exp \left(-E / E_{o}\right)$, where $\mathrm{j}$ is the differential intensity at energy $E$ in $\mathrm{MeV}$ nuc $^{-1}, j_{o}$ is the normalization constant, $\gamma$ is power-law spectral index, and $E_{o}$ is the e-folding energy [62]. Solid lines in panel (b) are fits to the spectra of the form $j_{=} j_{o} E^{-\gamma}$. It is noteworthy that the 0.03-3 MeV nuc ${ }^{-1} \mathrm{He}$ energy spectra associated with SIRs for events \#1 and \# 6 at 0.35 and $0.85 \mathrm{AU}$ respectively are identical - they behave as flat power laws modulated by exponential rollovers with e-folding energies of $\sim 0.4 \mathrm{MeV} \mathrm{nuc}^{-1}$. [61] argue that their new results provide evidence that suprathermal He ion observed in six CIR/SIR events by IS $\odot I S$ originate from sources or shocks beyond the location of PSP rather than from acceleration processes occurring at the locally observed compression regions (e.g. [19]). They also suggest that rarefactions regions that typically follow the SIRs may facilitate easier particle transport throughout the inner heliosphere, such that low-energy ions do not undergo as significant energy losses due to adiabatic deceleration, as has been predicted by existing models ([18], [63]). Finally, [61] remarked that the PSP observations they report pose a serious challenge for current EP transport models that predict turnovers in the energy spectra at energies below $\sim 0.5 \mathrm{MeV} n u c^{-1}$ due to adiabatic energy losses.

[64] further analyzed the $\mathrm{p}^{+}$enhancement that was observed by PSP on 2018, DOY 320 at 0.34 AU from the Sun. Figure 17, left provides an overview of the $\mathrm{p}^{+}$enhancement, which began on DOY 320, 2018 and persisted for almost four days. The event has been interpreted as being an SIR event ([28], [58], [61]) related to the compression region that passed over the s/c one day prior to the event onset. The event has a gradual onset and decline and shows little evidence of anisotropy or dispersion, consistent with most observations of SIR-related events. [28] identified the small, very low energy enhancement observed by EPI-Lo at the passage of the SIR on DOY 319 (see Figure 3) as being evidence of local acceleration at a weak compression. [64] divided the event into 5 intervals containing equal counts measured by EPI-Lo and determined how the

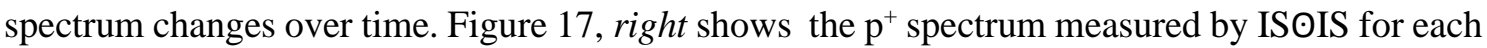
interval shown in Figure 17, left as well as the total spectrum for the entire period. Except for interval 1, fit by a simple power law, each spectrum was fit with a power law with an exponential rollover function. The fits were obtained using the same fitting routine as [61]. Figure 17, right shows a significant difference between the observed energetic particle spectrum during the first 

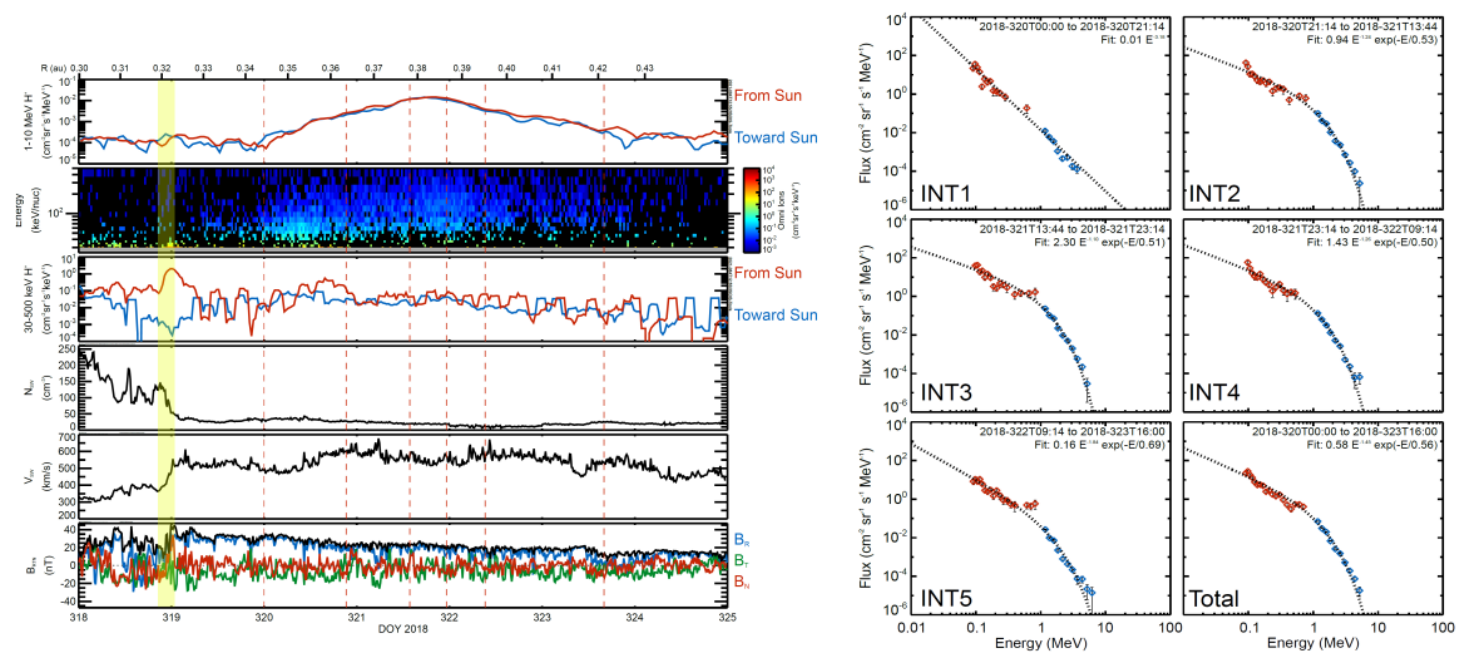

Figure 17. Analysis of an SIR associated $\mathrm{p}^{+}$event observed by PSP at 0.34 AU. From [64].

interval and the rest of the event. The first interval yields a spectrum that is close to a simple power law across the full energy range. After this interval, the spectrum becomes more curved, consistent with acceleration at a more distant source region (as in Figure 6 of [65]). As [64] point out, within the theoretical framework of [18], an evolution of the spectrum that is consistent with increasing modulation as the source of the particles becomes more distant later in the event would be expected to be seen. Instead, a spectrum that is quite constant after the first day of the event is observed indicating the modulation of EPs during transport is much weaker than predicted by [18], since the distance to the source region should be increasing during this time, increasing the transport effects on the particles. This is notably demonstrated by the relatively constant spectrum of low energy particles during the event, particularly intervals 2, 3 and 4, which should be the most affected by modulation due to lower mean free paths ([18], [65]).

The apparent lack of modulation also indicates that the magnetic field connecting the s/c to the source region deviates significantly from the usual Parker spiral orientation. A possible explanation may be that the local structure of the magnetic field is in a sub-Parker spiral configuration as described by [66] \& [67] in which the field becomes much more radial than usual ([68], [69]). This configuration arises as a result of magnetic footpoints moving across the coronal hole boundary, causing the field line to be stretched across the compression region between the fast and slow solar wind streams. This results in a large-scale field orientation that is much less transverse than the nominal Parker spiral, as presented in Figure 18, left from the companion paper by [70] which provides a theoretical framework explaining the radial field configurations that are often observed in the rarefaction regions that follow SIRs. Figure 18, right shows results by [70] who carried out EP transport modeling for this event. A comparison between observed (blue data points) and modeled (curves) differential fluxes at higher and lower energies is shown [70]. Modeled EPs are accelerated at the SIR reverse compression or shock and propagate back to PSP along sub-Parker field lines (black curves) and Parker field lines (blue curves). The sub-Parker magnetic field configuration provides a much shorter connection to the SIR, and explains why IS $\odot I S$ observes EP enhancements long after the stream interface passage. Based on their modeling analysis and the evidence shown by [64], [70] conclude that this event is best explained by acceleration occurring over a broad source region along the compression combined with a closer 

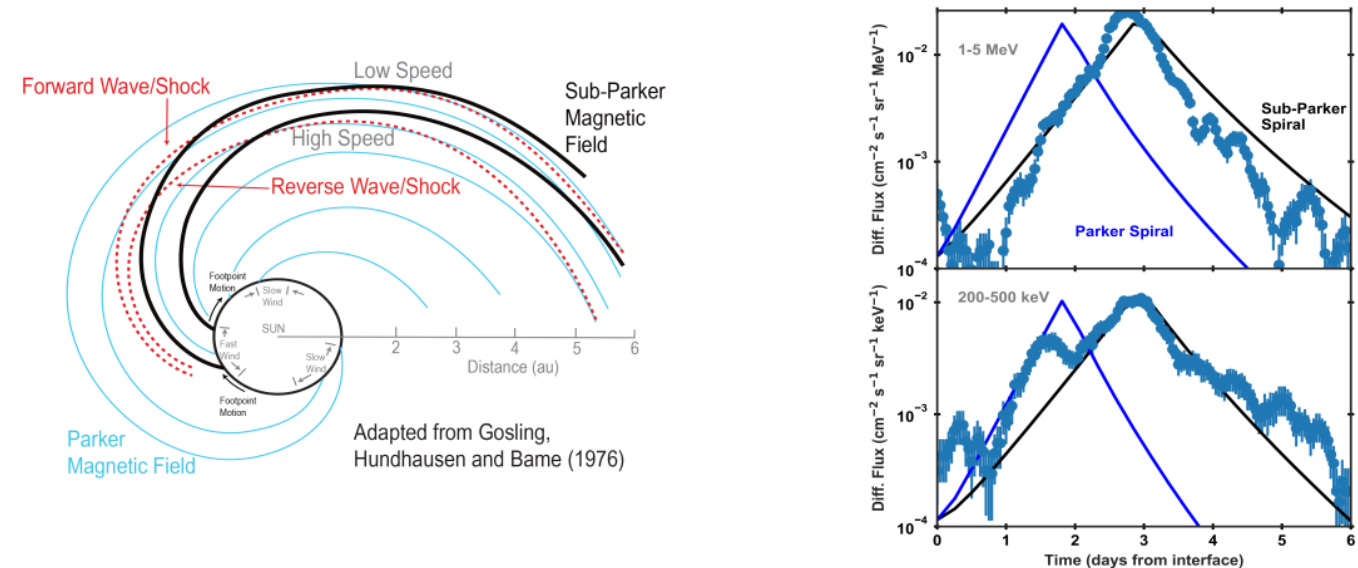

Figure 18. (left) With footpoint motion, IMF lines (black) connected between fast \& slow solar wind form the Sub-Parker spiral in rarefaction regions where the fast solar wind stretches out IMF lines in the radial direction (right) Model results compared to observations. From [70].

connection of PSP to this region as a result of the sub-Parker structure of the magnetic field. The time history of EP fluxes observed by ISOIS provides a new view of particle acceleration at SIRs throughout the inner heliosphere [70].

SolO observations provided a new addition to the study of the radial dependence of CIRassociated suprathermal ions in the inner heliosphere. Figure 19, top presents an overview of 226$320 \mathrm{keV} /$ nuc ions $\left(\mathrm{H},{ }^{4} \mathrm{He}, \mathrm{C}, \mathrm{O}\right)$ during the first orbit of SolO from 2020 April 15 through 2020

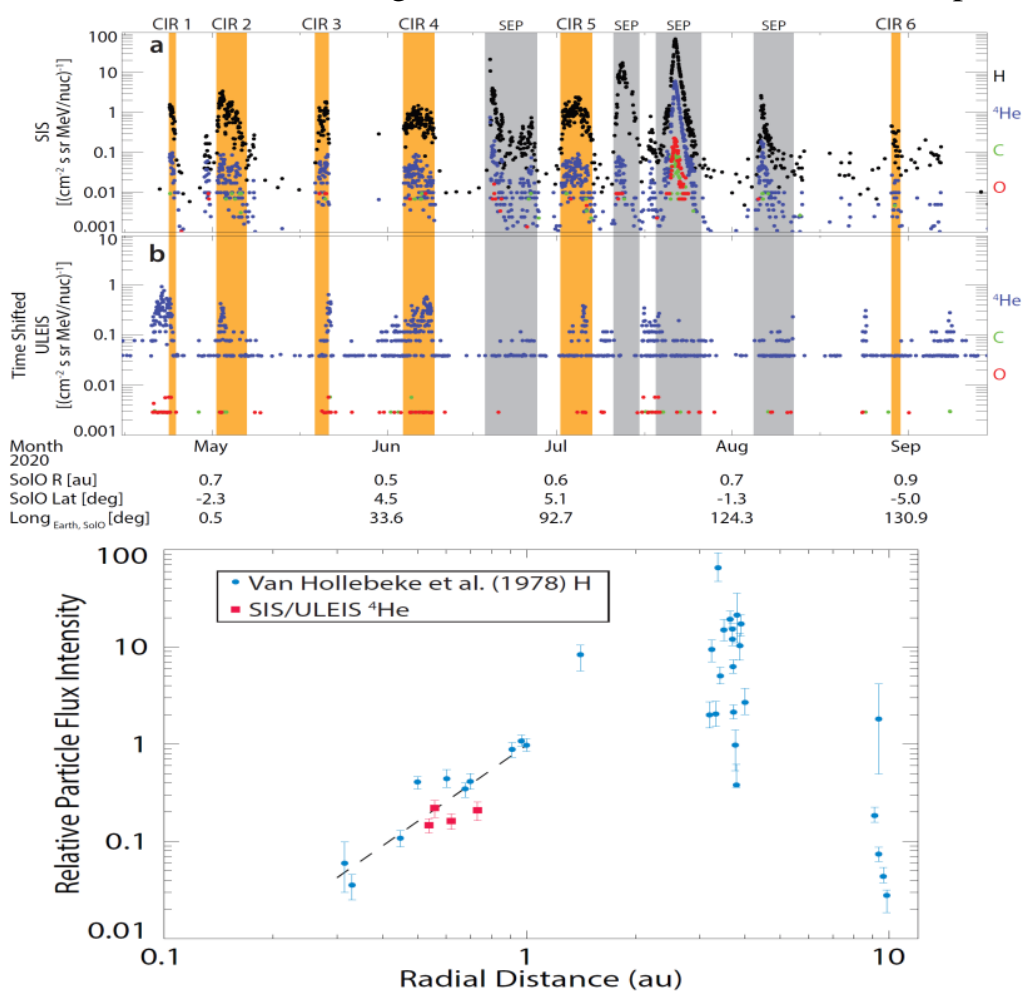

Figure 19. (top) Overview of 226-320 keV/nuc SolO/SIS and ACE/ULEIS observations from 2020 April 15 until September 15 (bottom) Relative particle flux intensity (normalised to intensity at $1 \mathrm{AU}$ ) as a function of radial distance from both SIS-ULEIS (red, 226-320 $\mathrm{keV} / \mathrm{nuc}{ }^{4} \mathrm{He}$ ) and from [21] (blue, 0.9-2.2 MeV H). Adapted from [46]. 
September 15 by the SolO/EPD/SIS instrument and the Ultra-Low Energy Isotope Spectrometer (ULEIS) instruments onboard ACE [46]. Six CIR-associated suprathermal EP events were seen (orange time period) along with four SEP events (gray time periods). [46] applied a wellcomputed time-shift of the ULEIS observations to the SIS observations for a better comparison. As shown in Figure 19, CIR-events 1-5 are all observed by both SIS and ULEIS. CIR event 6 was not observed by ULEIS, possibly due to it being the weakest of the CIR events observed by SolO or due to differences in latitude. [46] compared the ${ }^{4} \mathrm{He}$ spectra from ACE and SolO and found that the spectral slopes show general agreement, consistent with the PSP observations that investigated transport effects on inner heliospheric CIR-associated EP event spectra ([64], [71]). The turnover in the low energy part of the spectra as predicted by [18] was not observed. [46] also concluded that the radial gradients in the ${ }^{4} \mathrm{He}$ intensities between SIS and ULEIS are very similar to those found for more energetic $\mathrm{H}$ between Helios 1 and 2 and IMP-7 [21] (Figure 19, bottom).

\section{Cosmic Ray Observations in the Inner Heliosphere}

\subsection{Galactic Cosmic Rays (GCRs)}

[72] identified the first recurrent GCR decrease at SolO. Figure 20, top shows the GCR intensity as measured by the $>17-20 \mathrm{MeV}$ count rates of the EPD/HET detector from 16-24 June 2020 (black line) with an initial short-term GCR intensity increase and two consecutive decreases of different amplitudes observed. The first one starts at 00:00 UT on 18 June with an hourly percentage variaton of $\sim 4 \%$, whereas the second decrease, starts at 22:00 UT on 18 June (solid vertical line) with a variation of $\sim 2 \%$. The SolO/RPW derived solar wind speed (V) and density (n) are shown in the second and third panels of Figure 20 (black traces), respectively, whereas the black trace on the bottom panel shows the magnetic field intensity by SolO/MAG. A new magnetic field increase starting at 22:00 UT on 18 June, accompanied by an increase of $\mathrm{V}$ and later a decrease of $n$, suggest that a HSS, swept over SolO.

Figure 20. From top to bottom: Time profile of the HET normalized count rates, solar wind velocity, density and magnetic field magnitude for the period from 09:36 UT on 16 June to 09:35 UT on 24 June (black lines) and from 00:00 UT on 19 July to 00:00 UT on 27 July (green lines). The time difference between the two periods is 32.6 days. The black vertical line indicates the start of the second GCR depression at 22:00 UT on 18 June. From [72].

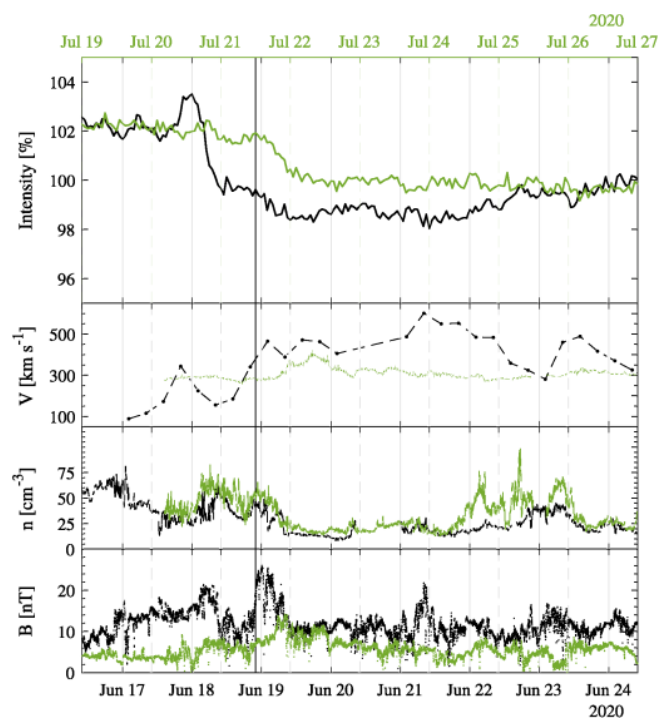

Calculations show that the GCR decrease observed to begin at 12:00 UT on 21 July (top panel, green line in Figure 20) is in very good agreement with the estimated recurrence time for the HSS. Recurrence of the same CIR in previous solar rotations is found to almost coincide with the time of corotating suprathermal ion events identified by [46] as CIR 1 and CIR 3 (see Figure 19). [73] present an interesting comparison of FDs at SolO and Earth using a multi-s/c approach. 


\subsection{Anomalous Cosmic Rays (ACRs)}

[74] presented the first observations of ACRs in to $36 \mathrm{Rs}$ (0.166 AU), focusing especially on helium as observed by PSP/IS $\odot I S$ (Orbits 1-3). A strong radial gradient of $\sim 25 \pm 5 \%$ /AU over energies of $\sim 4$ to $\sim 45 \mathrm{MeV} /$ nuc is derived by applying three methods to the detrended, eventsubtracted helium fluxes for the LET1 and HETB telescopes (Figure 21, left). These values are higher than all prior He observations further out in the heliosphere and come at a unique time in the understanding and modeling of EP transport and acceleration, particularly as both Voyagers have crossed the heliopause and IBEX has accumulated a full solar cycle of observations. Figure 21, right presents simulated radial gradients of ACR helium in the equatorial plane as a function of kinetic energy for varying distances in the heliosphere using the model of [75] with some minor updates and modifications. Relatively good agreement with results at $1 \mathrm{AU}$ is shown but as discussed by [74] their observations challenge considerably current transport models.
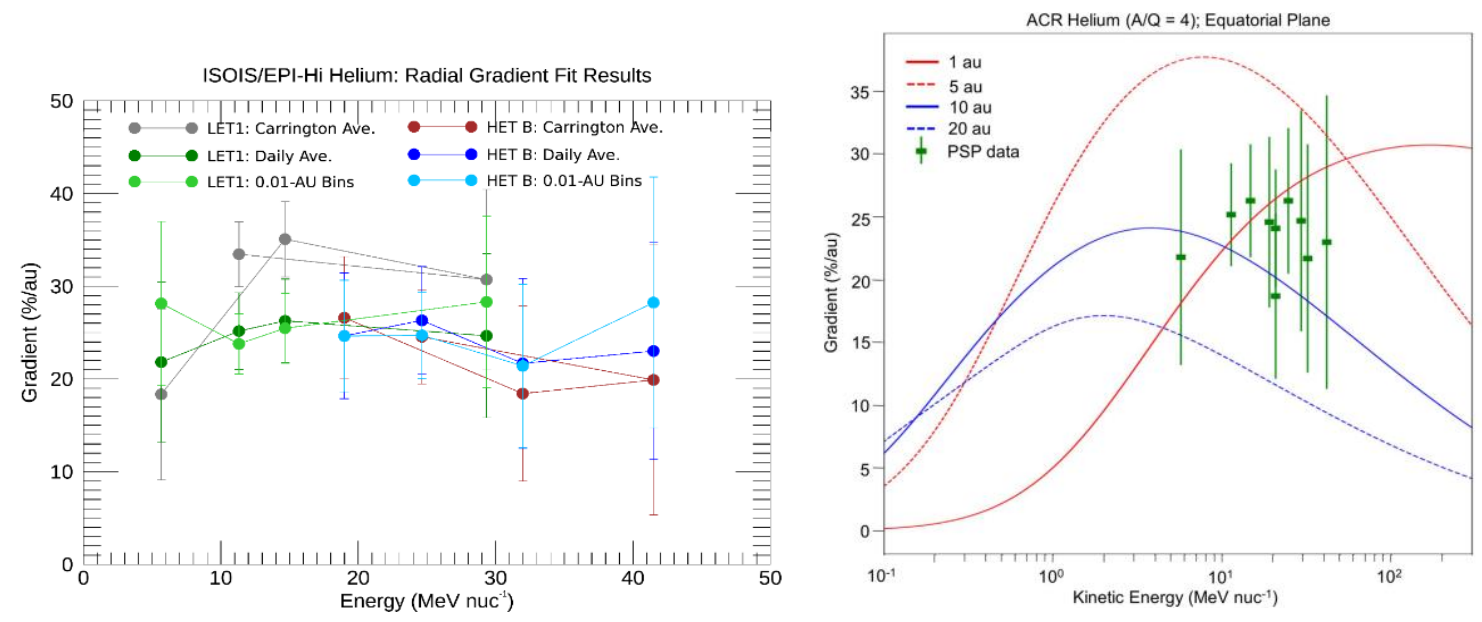

Figure 21. (left) Radial gradients derived by applying three methods to ISఠIS/EPI-Hi detrended, event-subtracted He fluxes for LET1 and HET B (right) Simulated radial gradients of ACR helium in the equatorial plane as a function of kinetic energy for radial distances of $1 \mathrm{AU}$ (solid red line), 5 AU (dashed red line), $10 \mathrm{AU}$ (solid blue line), and $20 \mathrm{AU}$ (dashed blue line). Values derived from PSP observations (green) compare relatively well with the values modeled at Earth. Results are generated using the model of [75]. Adapted from [74].

An important aspect to be considered, as [74] highlighted, is the unusual properties of the magnetic field near the Sun, with the radial component dominating and thus CRs should gain better access closer to the Sun as evidenced by their observations. Not only the Parker spiral should be considered but also the evidence for Sub- and Super-Parker deviations providing a magnetic connection of field lines across source regions of slow and fast solar wind, may be also important for modelling CR transport near the Sun and in the inner heliosphere [66], [67].

\section{Acknowledgements}

D.J.M and N.A.S were supported as a part of the Integrated Science Investigation of the Sun instrument suit on the NASA Parker Solar Probe Mission (contract NNN06AA01C). 


\section{References}

[1] H. Kunow, Energetic particles in the inner solar system, in Physics of the Inner Heliosphere, 2 Particles, Waves and Turbulence, Springer-Verlag 1991.

[2] G. Simnett, Energetic Particles in the Heliosphere, ASSL, Springer, 2017.

[3] I. G. Richardson, Energetic particles and corotating interaction regions in the solar wind, Space Sci. Rev., 111, 267, 2004.

[4] I. G. Richardson, Solar wind stream interaction regions throughout the heliosphere, Living reviews in solar phys., 15, 1, 2018.

[5] J. L. Freiherr von Forstner, J. Guo, R. F. Wimmer-Schweingruber et al., Using Forbush decreases to derive the transit time of ICMEs propagating from 1 AU to Mars, J. Geophys. Res: Space Physics, $123,39,2018$.

[6] J. L. Freiherr von Forstner, J. Guo, R. F. Wimmer-Schweingruber et al., Tracking and validating ICMEs propagating toward Mars using STEREO Heliospheric Imagers combined with Forbush Decreases detected by MSL/RAD, Space Weather, 17, 586, 2019.

[7] J. L. Freiherr von Forstner, J. Guo, R. F. Wimmer-Schweingruber et al., Comparing the properties of ICME-induced Forbush Decreases at Earth and Mars, J. Geophys. Res: Space Physics, 125, e2019JA027662, 2020.

[8] O. Witasse, B. Sánchez-Cano, M. L. Mays et al., Interplanetary coronal mass ejection observed at STEREO-A, Mars, comet 67P/Churyumov-Gerasimenko, Saturn, and New Horizons en route to Pluto: Comparison of its Forbush decreases at 1.4, 3.1, and 9.9 AU, J. Geophys. Res.: Space Physics, 122, 7865, 2017.

[9] R. M. Winslow, N. A. Schwadron, N. Lugaz et al., Opening a window on ICME-driven GCR modulation in the inner solar system, Astrophys. J., 856, 139, 2018.

[10] D. J. McComas and N. A. Schwadron, An explanation of the Voyager paradox: Particle acceleration at a blunt termination shock, Geophys. Res. Lett., 33, 2006.

[11] C. M. S. Cohen, Observations of Energetic Storm Particles: An Overview, in Solar Eruptions and Energetic Particles, Geophysical Monograph Series 165, American Geophysical Union, 2006.

[12] M. Desai and J. Giacalone, Large gradual solar energetic particle events, LRSP, 13, 3, 2016.

[13] O. E. Malandraki and N. B. Crosby, Solar Energetic Particles and Space Weather: Science and Applications, in Solar Particle Radiation Storms Forecasting and Analysis, vol. 444, Springer International Publishing, 2018a.

[14] O. E. Malandraki and N. B. Crosby, The HESPERIA HORIZON 2020 Project and Book on Solar Particle Radiation Storms Forecasting and Analysis, Space Weather, 16, 591, 2018b.

[15] C. M. S. Cohen, G. Li, G. M. Mason et al., Solar Energetic Particles, in Space Physics and Aeronomy Collection Volume 1: Solar Physics and Solar Wind, Geophysical Monograph 258, American Geophysical Union, 2021.

[16] J. Zhang, M. Temmer, N. Gopalswamy et al., Earth-affecting solar transients: a review of progresses in solar cycle 24, Progress in Earth and Planetary Science, 8, 56, 2021.

[17] G. Wibberenz and H. Cane, Multi-spacecraft observations of solar flare particle in the inner heliosphere, Astrophys. J. 650, 1199, 2006.

[18] L. A. Fisk and M. A. Lee, Shock acceleration of energetic particles in corotating interaction regions in the solar wind, Astrophys. J., 237, 620, 1980. 
[19] J. Giacalone, J. R. Jokipii, and J. Kóta, Particle acceleration in solar wind compression regions, Astrophys. J., 573, 845, 2002.

[20] J. H. Chen, N. A. Schwadron, E. Möbius et al, Modeling interstellar pickup ion distributions in corotating interaction regions inside 1 AU, J. Geophys. Res.: Space Physics, 120, 11, 9269, 2015.

[21] M. A. I. Van Hollebeke, F. B. McDonald, J. H. Trainor et al., The radial variation of corotating energetic particle streams in the inner and outer solar system, J. Geophys. Res.: Space Physics, 83, A10, 4723, 1978.

[22] N. J. Fox, M. C. Velli, S. D. Bale et al., The Solar Probe Plus mission: Humanity's First Visit to our Star, Space Sci. Rev., 204, 7, 2016.

[23] D. J. McComas, N. Alexander, N. Angold et al., Integrated Science Investigation of the Sun (ISIS): Design of the Energetic Particle Investigation, Space Sci. Rev., 204, 187, 2016.

[24] M. E. Hill, D. G. Mitchell, G. B. Andrews et al., The Mushroom: A half-sky energetic ions and electron detector, J. Geophys. Res.: Space Physics, 122, 1513, 2017.

[25] M. E. Wiedenbeck, N. G. Angold, B. Birdwell et al., Capabilities and Performance of the HighEnergy Energetic-Particles Instrument for the Parker Solar Probe Mission, in proceedings of $35^{\text {th }}$ International Cosmic Ray Conference-ICRC2017, PoS(ICRC2017)016, 35, 16.

[26] D. Müller, O. C. St. Cyr, I. Zouganelis et al., The Solar Orbiter mission, Science Overview, A\&A, 642, A1, 2020.

[27] J. Ródriguez-Pacheco, R. F. Wimmer-Schweingruber, G. M. Mason et al., The Energetic Particle Detector, Energetic particle instrument suite for the Solar Orbiter mission, A\&A, 642, A7, 2020.

[28] D. J. McComas, N. Alexander, N. Angold et al., Probing the energetic particle environment near the Sun, Nature, 576, 223, 2019.

[29] S. D. Bale, S. T. Badman, J. W. Bonnell et al. Highly structured slow solar wind emerging from an equatorial coronal hole, Nature, 576, 237, 2019.

[30] J. Giacalone, D. G. Mitchell, R. C. Allen et al., Solar Energetic Particles produced by a slow Coronal Mass Ejection at 0.25 AU, Astrophys. J. Suppl. Ser., 246, 29 (12pp), 2020.

[31] E. N. Parker, The passage of energetic charged particles through interplanetary space, Planet. And Space Sci., 13, 9, 1965.

[32] D. G. Mitchell, J. Giacalone, R. C. Allen et al., CME-associated energetic ions at 0.23 AU: Consideration of the auroral pressure cooker mechanism operating in the low corona as a possible energization process, Astrophys. J. Suppl. Ser., 246, 59 (8pp), 2020.

[33] R. A. Leske, E. R. Christian, C. M. S. Cohen et al., Observations of the 2019 April 4 Solar Energetic Particle event at the Parker Solar Probe, Astrophys. J. Suppl. Ser., 246, 35 (10pp), 2020.

[34] D. V. Reames, Element Abundances in Solar Energetic Particles and the solar corona, Sol. Phys., 289, 977, 2014.

[35] J. M. Laming and U. Feldman, The solar Helium abundance in the outer corona determined from observations with SUMER/SOHO, Astrophys. J., 546, 552, 2001.

[36] N. A. Schwadron, S. Bale, J. Bonnell et al., Seed population preconditioning and acceleration observed by the Parker Solar Probe, Astrophys. J. Suppl. Ser., 246, 33 (17pp), 2020.

[37] O. E. Malandraki, E. T. Sarris, L. J. Lanzerotti et al., Solar energetic particles inside a coronal mass ejection event observed with the ACE spacecraft, J. Atmos. \& Sol.-Terr. Phys., 64, 517, 2002. 
[38] M. Wiedenbeck, R. Bučík, G. M. Mason et al., ${ }^{3}$ He-rich solar energetic particle observations at the Parker Solar Probe and near Earth, Astrophys. J. Suppl. Ser., 246, 42 (15pp), 2020.

[39] G. Livadiotis and D. J. McComas, Beyond kappa distributions: Exploiting Tsallis statistical mechanics in space plasmas, J. Geophys. Res.: Space Physics, 114, A11, 2009.

[40] G. Livadiotis and D. J. McComas, Exploring transitions of space plasmas out of equilibrium, Astrophys. J., 714, 971, 2010.

[41] C. J. Joyce, D. J. McComas, N. A. Schwadron et al., Energetic particle evolution during coronal mass ejection passage from 0.3 to $1 \mathrm{AU}, \mathrm{A} \& \mathrm{~A}, 651, \mathrm{~A} 2,2021$.

[42] C. M. S. Cohen, E. R. Christian, A. C. Cummings et al., Parker Solar Probe observations of He/H abundance variations in SEP events inside 0.5 AU, A\&A, 650, A23, 2021.

[43] R. Chhiber, W. H. Matthaeus, C. M. S. Cohen et al., Magnetic field line random walk and solar energetic particle path lengths, Stochastic theory and PSP/ISOIS observations, A\&A, 650, A26, 2021.

[44] R. F. Wimmer-Schweingruber, N. P. Janitzek, D. Pacheco et al., First year of energetic particle measurements in the inner heliosphere with Solar Orbiter's Energetic Particle Detector, A\&A, 656, A22, 2021 (SO Cruise Phase SI).

[45] G. M. Mason, G. C. Ho, R. C. Allen et al., Quiet-time low energy ion spectra observed on Solar Orbiter during solar minimum, A\&A, 656, L5, 2021 (SO Cruise Phase SI).

[46] R. C. Allen, G. M. Mason, G. C. Ho et al., Suprathermal particles from corotating interaction regions during the first perihelion pass at Solar Orbiter, A\&A, 656, L2 2021 (SO Cruise Phase SI).

[47] R. Gómez-Herrero, D. Pacheco, A. Kollhoff et al., First near-relativistic solar electron events observed by EPD onboard Solar Orbiter, A\&A, 656, L3, 2021 (SO Cruise Phase SI).

[48] M. Maksimovic, S. D. Bale, T. Churst et al., The Solar Orbiter Radio and Plasma Waves (RPW) instrument, A\&A, 642, A12, 2021.

[49] O. E. Malandraki, N. Agueda, A. Papaioannou, Scientific analysis within SEPServer - New perspectives in solar energetic particle research: The case study of the 13 July 2005 event, Sol. Phys. $281,333,2012$.

[50] R. Bučík, ${ }^{3} \mathrm{He}$-rich solar energetic particles: solar sources, Space Sci. Rev., 216, 24, 2020.

[51] D. V. Reames, Solar Energetic Particles, 978, Springer Cham., 2021.

[52] G. M. Mason, G. C. Ho, R. C. Allen et al., ${ }^{3}$ He-rich solar energetic particle events observed on the first perihelion pass of Solar Orbiter, A\&A, 656, L1, 2021.

[53] R. Bučík, G. M. Mason, R. Gómez-Herrero et al., The long period of ${ }^{3} H e$-rich solar energetic particles measured by Solar Orbiter 2020 November 17-23, A\&A, 656, L11, 2021.

[54] A. Kollhoff, A. Kouloumvakos, D. Lario et al., The first widespread solar energetic particle event observed by Solar Orbiter on 2020 November 29, A\&A, 656, A20, 2021.

[55] T. Laitinen, and S. Dalla, From sun to interplanetary space: what is the pathlength of Solar Energetic Particles?, Astrophys. J., 887, 222, 2019.

[56] A. Kouloumvakos, R. Y Kwon, L. Rodríguez-García et al., The first widespreas solar energetic particle event of solar cycle 25 on 2020 November 29. Shock wave properties and the wide distribution of SEPs, A\&A, in press, 2022. 
[57] I. G. Richardson, T. T. von Rosenvinge, H. Cane et al., > 25 MeV proton events observed by the high energy telescopes on the STEREO A and B spacecraft and/or at Earth during the first $\sim$ seven years of the STEREO mission, Sol. Phys., 289, 3059, 2014.

[58] C. M. S. Cohen, E. R. Christian, A. C. Cummings et al. Energetic particle increases associated with Stream Interaction Regions, Astrophys. J. Suppl. Ser., 246, 20, 2020.

[59] R. C. Allen, D. Lario, D. Odstrcil et al. Solar wind streams and Stream Interaction Regions observed by the Parker Solar Probe with corresponding observations at 1 AU, Astrophys. J. Suppl. Ser., 246, $36,2020$.

[60] I. G. Richardson, L. M. Barbier, D. V. Reames et al., Corotating MeV/amu ion enhancements at $\leq 1$ AU from 1978 to 1986, J. Geophys. Res., 98, 13, 1993.

[61] M. I. Desai, D. G. Mitchell, J. R. Szalay et al., Properties of suprathermal-through-energetic He ions associated with Stream Interaction Regions observed over the Parker Solar Probe's first two orbits, Astrophys. J. Suppl. Ser., 246, 56, 2020.

[62] F.C. Jones and D.C. Ellison, The plasma physics of shock acceleration, Space Sci. Rev., 58, $259,1991$.

[63] L. Zhao, G. Li, R. W. Ebert et al., Modeling transport of energetic particles in corotating interaction regions: A case study, J. Geophys. Res.: Space Physics, 121, 77, 2016.

[64] C. J. Joyce, D. J. McComas, N. A, Schwadron et al., Time evolution of stream interaction region energetic particle spectra in the inner heliosphere, A\&A, 650, L5, 2021.

[65] G. M. Mason, R. von Steiger, R. B. Decker et al. Origin, Injection and Acceleration of CIR particles: Observations, in Corotating Interaction Regions, Dodrecht, Springer, Netherlands, 327, 1999.

[66] N. A. Schwadron and D. J. McComas, The sub-Parker spiral structure of the heliospheric magnetic field, Geophys. Res. Lett., 32, L03112, 2005.

[67] N. A. Schwadron and D. J. McComas, Switchbacks explained: Super-Parker fields- The other side of the sub-Parker spiral, Astrophys. J., 909, 95, 2021.

[68] N. A. Schwadron, D. J. McComas, H. E. Elliott et al., Solar wind from coronal hole boundaries, J. Geophys. Res.: Space Physics, 110, A04104, 2005.

[69] N. A. Schwadron, An explanation of strongly underwound magnetic field in co-rotating rarefaction regions and its relationship to footpoint motion on the sun, Geophys. Res. Lett., 29, 1663, 2002.

[70] N. A. Schwadron, C. J. Joyce, A. Aly et al., A new view of energetic particles from stream interaction regions observed by Parker Solar Probe, A\&A, 650, A24, 2021.

[71] R. C. Allen, G. C. Ho, G. M. Mason et al., Radial evolution of a CIR: Observations from a nearly radially aligned event between Parker Solar Probe and STEREO A, Geophys. Res. Lett, 48:e91376, 2021.

[72] A. Aran, D. Pacheco, M. Laurenza et al., Evidence for local acceleration in the first recurrent galactic cosmic ray depression observed by Solar Orbiter, The ion event on 19 June 2020, A\&A, 656, L10, 2021.

[73] J. L. Freiherr von Forstner, M. Dumbović, C. Möstl et al., Radial evolution of the April 2020 sleath coronal mass ejection between 0.8 and 1 AU - Comparison of Forbush decreases at Solar Orbiter and near the Earth, A\&A, 656, A1, 2021.

[74] J. S. Rankin, D. J. McComas, R. S. Leske et al., First observations of Anomalous Cosmic Rays in to 36 Solar Radii, Astophys. J., 912, 139, 2021.

[75] R. D. Strauss and M. S. Potgieter, Modeling anomalous cosmic ray oxygen gradients over successive solar cycles, J. Geophys. Res, 115, A12111. 